Peter Auer (Freiburg i.Br.)

\title{
Ethnische Marker im Deutschen zwischen Varietät und Stil ${ }^{*}$
}

\begin{abstract}
Der Beitrag zeichnet im ersten Teil die historische Entwicklung ethnischer und polyethnischer Sprechweisen im Deutschen nach und skizziert die verschiedenen Transformationen ihrer indexikalischen Bedeutung. Im zweiten Teil des Beitrags wird anhand von Daten aus Stuttgart diskutiert, ob es sich bei den heute verwendeten polyethnischen Markern um Komponenten eines sozialen Stils oder um eine emergente neue Varietät handelt.
\end{abstract}

\section{Einleitung}

Das Thema dieses Bandes - das „Deutsch der Migranten“ - ist voller terminologischer und konzeptioneller Fallen. Viele der Bezeichnungen, die wir verwenden, um auf die Sprecher und Sprecherinnen zu verweisen, über die wir wissenschaftliche Aussagen machen wollen (,Migrant/inn/en', ,Menschen mit Migrationshintergrund ${ }^{\circ}$ ), und viele der linguistischen Termini, die wir benutzen, um einen Teil ihres Repertoires an sprachlichen Ausdrucksmitteln zu erfassen (,Ethnolekt', ,Dialektt, ,Multiethnolekt'), sind problematisch und diskussionsbedürftig. Worum es geht, sind die sprachlichen Konsequenzen der weltweiten, in den letzten 20 Jahren stark angewachsenen Migrationsbewegungen in die ökonomisch prosperierenden Gebiete der Welt, besonders in die Großstädte in Europa, Nordamerika, Asien und Australien, aus wirtschaftlich und/oder politisch bedrohten Gebieten an der (relativen) Peripherie. Die genauen demografischen, sozialen und linguistischen Parameter dieser Migrationsbewegungen sind von Fall zu Fall verschieden, sie haben aber in New York, London oder Paris, Hongkong, Singapur oder Melbourne genauso wie in Berlin, Hamburg oder Stuttgart zur Herausbildung hochdifferenzierter, für die Linguistik neuer Sprachkontaktsituationen geführt (superdiversity; Vertovec 2007). Der Sprachwissenschaft gelingt es nur allmählich, die Grundzüge einer Soziolinguistik der Globalisierung (Blommaert 2010) zu entwickeln, die diesen Entwicklungen theoretisch und methodisch Rechnung trägt und die die grundsätzliche Entkoppelung von Sprache, Individuum und geografischem Raum, die damit einher geht, erfassen kann (Quist 2010). Insbesondere die Soziolinguistik steht vor einer Herausforderung. Die Entwicklung verläuft - etwa im Vergleich mit

Für Kommentare und Verbesserungsvorschläge danke ich Vanessa Siegel und Arnulf Deppermann. 
der so genannten Gastarbeiterimmigration und deren sprachlichen Folgen - rasant schnell und will überdies nicht so recht in die bekannten und beschriebenen Muster passen. Das liegt auch daran, dass im Mittelpunkt des soziolinguistischen Interesses heute nicht erwachsene Migranten stehen, die mehr oder weniger erfolgreich die Varietät des Aufnahmelands als Zweitsprache erwerben, sondern bereits in Deutschland geborene und/oder aufgewachsene Kinder und Jugendliche, deren sprachlich-stilistische Selbstverortung im sozialen Raum sich an vielfältigen Mustern ausrichtet, biografisch instabil und situativ variabel ist und durch die permanenten Veränderungen der demografischen und sprachlichen Strukturen des sozialen Umfelds (z.B. durch neue Immigrationswellen) ständig Neuorientierungen erfordert. Nicht zuletzt stehen diese jugendlichen Sprechweisen in einem Spannungsfeld zwischen medialer Stilisierung und alltäglichem Gebrauch.

Die Forschung über die Folgen der Immigration für die urbanen jugendsprachlichen Sprechweisen sind eingebettet in politische Diskussionen um angebliche Parallelgesellschaften in Ausländerghettos; zugleich finden sie (seit 2001) im Kontext der Diskussion der Ergebnisse der Pisa-Studien statt, die den unterdurchschnittlichen Bildungserfolg von Jugendlichen aus Immigrantenfamilien dokumentiert haben. Schon dieses politische Umfeld der heutigen Diskussion zeigt, dass es nicht einfach um Jugendliche ,mit Migrationshintergrund' geht. Ebenso wenig ist Mehrsprachigkeit an sich das Problem. Die öffentliche Diskussion blendet in der Regel all die Jugendlichen mit Migrationshintergrund aus, die gut in den gesellschaftlichen mainstream eingebettet sind. Es geht ihr um die Ränder der Gesellschaft, um diejenigen Jugendlichen, die sich vielleicht selbst ironisch als ,Kanaken' bezeichnen, die in einem Stadtviertel wohnen, das sie u.U. ,Ghetto' oder ,Kiez' nennen, die sich zu einem guten Teil außerhalb der von, den Deutschen' beherrschten Gesellschaft und in diesem Sinn als ,Ausländer' fühlen (selbst wenn sie einen deutschen Pass haben), und die sich mit ihrer schulischen und beruflichen Karriere schwer tun, ob sie sich nun um sie bemühen oder nicht. Es geht also um gesellschaftliche Problemfelder.

Aus soziolinguistischer Perspektive stellen sich in Bezug auf diese Jugendlichen eine Reihe von Fragen, etwa:

Stimmt der in der Öffentlichkeit inzwischen vorherrschende Eindruck, dass sich ihre Sprache deutlich vom autochthonen Deutsch unterscheidet und innovative Merkmale aufweist, die weder im gesprochenen Deutsch sozial vergleichbarer, deutsch-monolingualer Gruppen von Jugendlichen vorkommen noch als lernersprachlich erklärt werden können?

Wo liegen diese Unterschiede, wie frequent sind sie und welchen Stellenwert haben sie im Bezug auf die Struktur des autochthonen Deutschen?

Gibt es andere sprachliche Unterschiede zu monolingual deutschsprachigen Jugendlichen, etwa bei der Verwendung von regionalen und dialektalen Formen? 
Können die Unterschiede als ethnische Marker bezeichnet werden, d.h. indizieren sie die Zugehörigkeit zu einer spezifischen ethnischen Gruppe, oder handelt es sich um poly- oder pan-ethnische Merkmale, die allgemein von Jugendlichen mit unterschiedlichen Migrationsgeschichten verwendet werden?

Verwenden auch Jugendliche mit monolingualem deutschen Sprachhintergrund solche sprachlichen Merkmale und was bedeutet es, wenn sie es tun?

Werden die sprachlichen Merkmale situationsspezifisch eingesetzt, d.h. sind sie Teil eines „ethnischen Repertoires“ (Sharma 2011; Benor 2010), das fallweise aktiviert und in den dafür nicht geeigneten Situationen durch die entsprechenden sprachlichen Ausdrucksformen der deutschen Mehrheitsgesellschaft ersetzt wird?

Schließlich: Schlägt sich die Verwendung der vom autochthonen Deutsch abweichenden Merkmale in schlechteren Schulleistungen nieder, ist sie mit diesen lediglich in einer nicht-kausalen Weise korreliert, oder haben die beiden Dinge überhaupt nichts miteinander zu tun?

Es fehlen uns immer noch belastbare empirische Untersuchungen, um auf all diese Fragen guten Gewissens Antworten zu geben. Lediglich über die Situation von Jugendlichen in Mannheim und Berlin wissen wir durch die Untersuchungen von Keim (z.B. 2007) und Wiese (2006, 2009, 2012; Wiese/Duda ersch.demn.) wenigstens in Ansätzen Bescheid und können deshalb zumindest zu einigen der genannten Fragen Antworten vorschlagen. Allerdings gibt es auch zu diesen Städten keine quantitativen Untersuchungen. Die Karriere dürftig dokumentierter und belegter, ${ }^{1}$ angeblich ghettodeutscher Strukturen wie ich mach dich Messer in den Medien und die Gefahren, die sich aus den semantischen Konnotationen des Beispiels und der in diesem Fall sehr erfolgreichen „Enkodierung“ (Agha 2003, vgl. Beginn des Abschnitts 2.2) der ghettodeutschen Varietät in der Öffentlichkeit ergeben ist beredtes Beispiel dafür, dass wir Linguisten uns nicht im Elfenbeinturm der Wissenschaft befinden.

Der vorliegende Beitrag hat zwei Ziele. Zum einen legt es die rapide Entwicklung der Phänomene nahe, von einer systematischen Darstellung zugunsten einer historischen abzusehen. Ich werde also zunächst die Geschichte des ,Ghettodeutschen ${ }^{2}$ skizzieren. Im zweiten Teil des Beitrags werden erste Ergebnisse einer neuen Untersuchung (poly-)ethnischer Sprechweisen in multiethnischen Jugendlichengruppen in Stuttgart vorgestellt.

\footnotetext{
Nach Wiese (2009) handelt es sich um kein Belegbeispiel, sondern ,a ritualised threat that is often quoted in public discussions of Kiezdeutsch".

2 Die Bezeichnungen ,Ghettodeutsch`, ,Straßensprache', ,Kiezdeutsch`werden im Folgenden austauschbar verwendet und dabei möglichst der Begriff benutzt, der von den untersuchten Jugendlichen in den verschiedenen Städten selbst gebraucht wird; es handelt sich also immer um die Ethnonyme, nie um wissenschaftliche Begriffe.
} 


\section{2. ,Türkendeutsch` - ,Kanaksprak` - ,Kiezdeutsch ${ }^{6}$ und so weiter: Skizze einer Entwicklung}

Um die komplexe Entwicklung ethnischer Sprechweisen in Deutschland in den letzten Jahrzehnten zu beschreiben, gehe ich von einem 2003 entwickelten Modell aus (Auer 2003). Allerdings verbietet es sich heute, die Begriffe Ethnolekte und Soziolekt noch mit derselben Selbstverständlichkeit zu verwenden wie damals, so dass ich eine revidierte Version vorschlage:

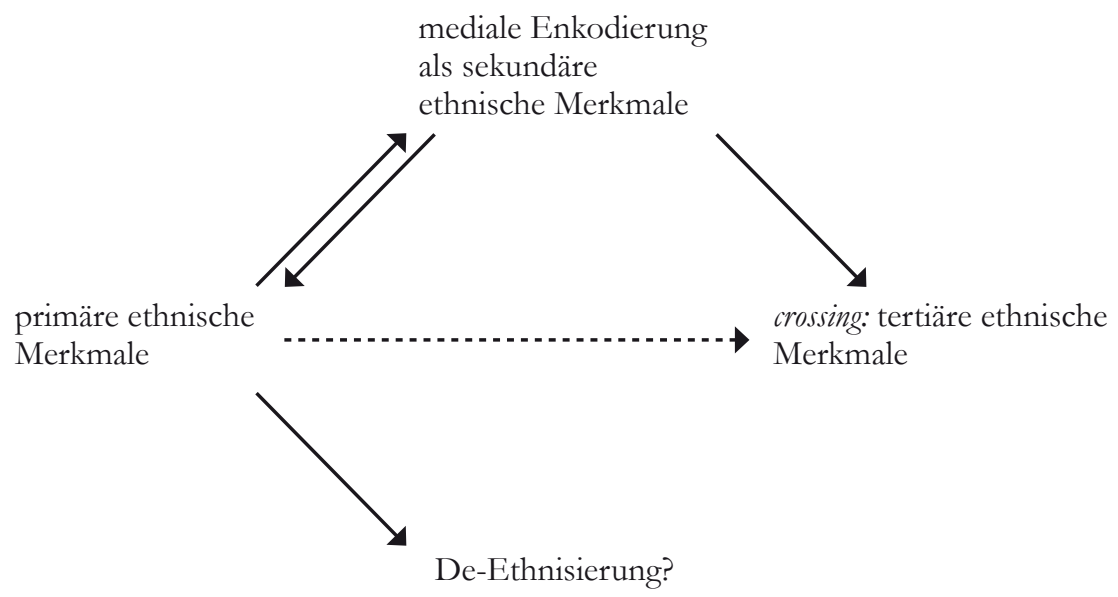

Abb. 1: Ein einfaches Modell ethnischer Merkmale im Deutschen (revidiert aus Auer 2003)

Ich werde im Folgenden die Stationen einer Entwicklung nachzeichnen, die sich in den Begriffen ,primär, sekundär, tertiär' niederschlägt.

2.1 Die Anfänge ethnischer jugendlicher Sprechweisen in türkisch-dominierten Milieus

Die ersten Belege für die Herausbildung ethnischer Sprechweisen, die sich nicht als lernersprachliche Strukturen aus dem ungesteuerten Erwerb des Deutschen als Zweitsprache erklären lassen, sondern sozial etablierte Sprechweisen (soziale Stile) unter Jugendlichen mit Migrationshintergrund waren, die in Deutschland geboren und aufgewachsen sind, stammen aus den frühen 90er Jahren und sind in Tertilts „Ethnographie einer Jugendbande“, den Frankfurter „Power Boys“, dokumentiert (Tertilt 1996). Die Tonaufnahmen dieser türkischstämmigen Jugendlichen zeigen bereits wesentliche Merkmale des heutigen ,Kiezdeutsch': 
(1) (Erzählung eines Mitglieds der „Turkish Power Boys“; vgl. Tertilt 1996; eigenes Transkript einer Aufnahme; ethnische Merkmale unterstrichen)

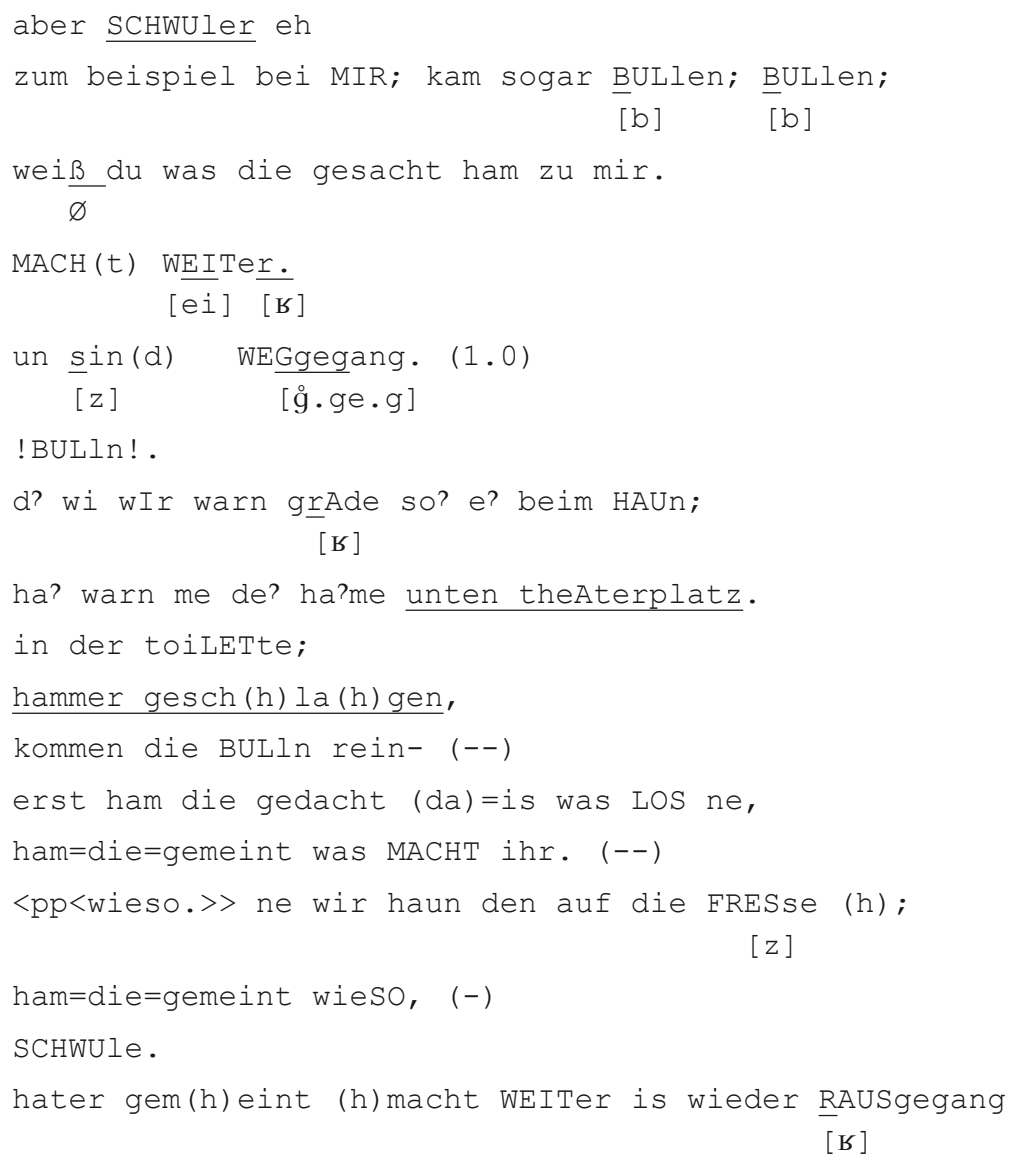

Auffällig ist der Sprecher vor allem in phonetischer Hinsicht; viele Merkmale wie Monophthongierungstendenzen bei den Diphthongen, teils starke Stimmhaftigkeit der Obstruenten, auslautende Clusterschwächungen sowie typische frikativische R-Qualitäten werden wir später auch bei den Stuttgarter Sprechern finden. In der Grammatik gibt es ein Beispiel für einen fehlenden indefiniten Artikel (erste Zeile), ein relativ unspektakuläres Beispiel für die Weglassung einer Präposition (unten [im/ am] Theaterplatz), die marginal auch im autochthonen Deutsch möglich erscheint, sowie evtl. die Weglassung eines Pronomens (hammer [ihn, einen] geschlagen). Daneben stehen 
allerdings auch zahlreiche Fälle für die standardkonforme Verwendung von Artikeln (die Bullen), Präpositionen (in der Toilette, auf die Fresse) und Anaphern (wir haun den auf die Fresse). Dialektale Färbungen sind hin und wieder erkennbar (im Ausschnitt etwa im stimmhaften intervokalischen Sibilanten bei der Artikulation von Fresse), aber nicht ausgeprägt. Die heute stereotypisierte Verwendung der Floskel ischwör(s) dient dem emphatischen Abschluss der Erzählung und fällt vor allem durch die vollständige Assimilierung des wortauslautenden an den wortanlautenden Frikativ zwischen Pronomen und Verb auf, die schon zu dieser Zeit auf eine Entwicklung zum univerbierten Diskursmarker hindeutet. Auch die aus dem späteren medialen Ethnolekt bekannten Floskeln korrekt und krass kommen bei den Turkish Power Boys übrigens schon vor.

Es scheint gerechtfertigt, hier von primären ethnischen Merkmalen zu sprechen, weil in dieser Phase Fragen der medialen Stilisierung und Kodierung wohl noch keine Rolle gespielt haben. Tertilts Analysen zufolge definierten sich die Turkish Power Boys durch ihre ethnische Zugehörigkeit, eben durch ihr Türkischsein. Es gab auch einige nicht-türkische Mitglieder der etwa 20-köpfigen Gang (ein Jugoslawe, ein Marokkaner, ein Italiener), die aufgrund ihrer Freundschaft mit anderen, türkischen Gruppenmitgliedern und wegen ihres Ausländerstatus akzeptiert wurden (Tertilt 1996, S. 24). Die Zugehörigkeit nicht-türkischer Gruppenmitglieder bedurfte nach Tertils Analysen aber besonderer sozialer Lizenzierungen.

Ethnische Sprechweisen unter Jugendlichen gibt es also seit mindestens 20 Jahren, tendenziell mit denselben Merkmalen wie heute. Das damalige massive quantitative Übergewicht der türkischstämmigen Jugendlichen in allen westdeutschen Großstädten und Berlin macht es extrem unwahrscheinlich, dass sie in anderen als eben diesen, dominant türkischen Netzwerken entstanden sind. Zu dieser Zeit bedeutet, Ausländer sein' in metonymischer Verengung in erster Linie, Türke sein'.

\subsection{Medialisierung: die „Enkodierung“ sekundärer ethnischer Merkmale}

Etwa zehn Jahre später (vgl. zu Hamburg Dirim/Auer 2004, zu Mannheim u.a. Keim 2007; außerdem die Sammlung v.a. von Hörbelegen aus Süddeutschland bei Füglein 2000) waren einige bedeutende Veränderung eingetreten. Die wichtigste war vielleicht, dass einige Merkmale der ethnischen jugendlichen Sprechweisen in dieser Zeit durch die Medien zu einem sekundären ethnischen Stil kodiert wurden - eine Entwicklung, die in den Mitt-1990er-Jahren begann (Beginn der Karriere von Erkan \& Stefan) und ca. 2005 ihren Höhepunkt fand. Der Begriff der Enkodierung wird hier als Übersetzung von enregisterment (Registrierung = Schaffung eines Registers; 
Enkodierung $=$ Schaffung eines Codes) vorgeschlagen, mit dem Agha (2003, 2007) Prozesse beschreibt, „whereby distinct forms of speech come to be socially recognized (or enregistered) as indexical of speaker attributes by a population of language users". Agha beschäftigt sich weniger mit dem tatsächlichen Sprachgebrauch, als mit den „models of language use that are disseminated along identifiable trajectories in social space" (2007, S. 38). Er analysiert die Prozesse der Typifizierung, durch die Konstellationen von linguistischen Merkmalen (die er Register nennt) soziale Bedeutung erhalten. Dies geschieht dadurch, dass diese Merkmalskonstellationen mit ebenfalls typisierten Sprechern assoziiert werden (role alignment). Die spezifische Reflexivität, die den Prozess der Enkodierung kennzeichnet, besteht also darin, dass zugleich soziale (Sprecher-)Typen und Codes geschaffen werden, die so aufeinander abgebildet werden können, dass sie als ihre natürlichen Entsprechungen erscheinen. Enkodierungsprozesse finden natürlich auch in der Alltagskommunikation statt, aber in spätmodernen Gesellschaften sind sie zumindest in Bezug auf jugendliche Sprechweisen kaum anders als durch mediale Vermittlung denkbar.

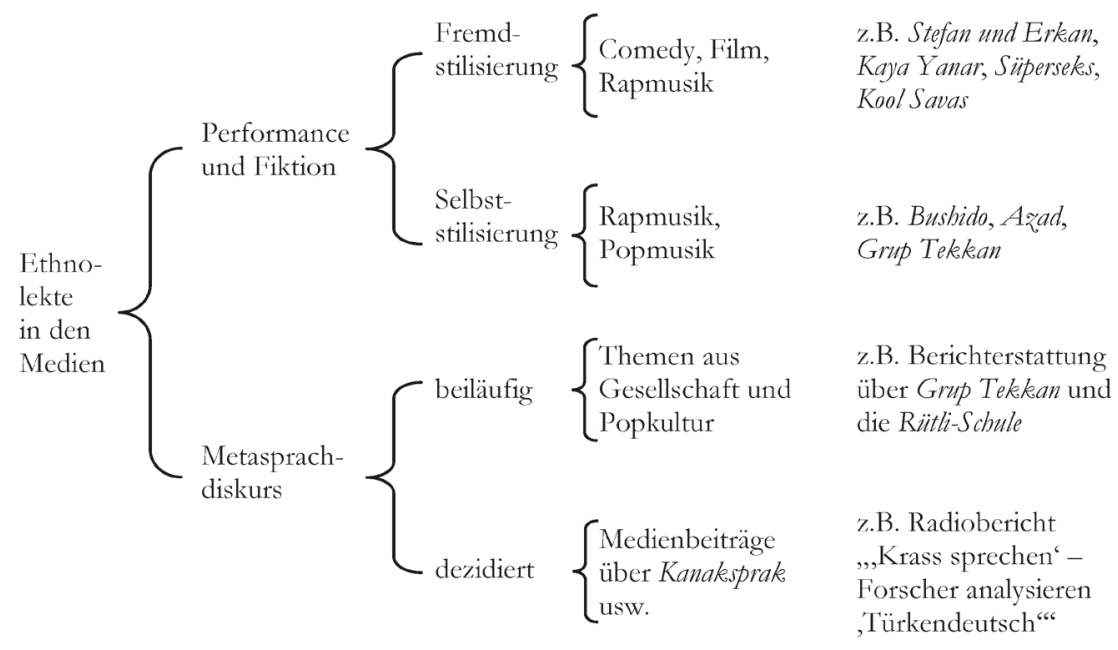

Abb. 2: Mediale Enkodierungsformen für ethnische Sprechweisen (aus: Androutsopoulos 2007)

Die Enkodierung ethnischer Stile in den Medien ist vielfältig untersucht worden, insbesondere in den Arbeiten von Androutsopoulos (z.B. 2007) (vgl. Abb. 2). Die Zuordnung von Sprechweisen zu bestimmten Typen jugendlicher Sprecher am Rand der Gesellschaft beruhte einerseits auf der Generalisierung von Merkmalen, die tatsächlich in ethnischen Stilen vorkommen (vgl. Bsp. 1), die aber forciert und generalisiert wurden, andererseits auf der Hinzunahme von Merkmalen, die frei hinzuerfunden wurden. 
Mehrere Enkodierungen konkurrierten miteinander (und tun es teils noch heute). Einerseits gab es die Comedy-Variante, die in Verbindung mit einem bestimmten Typ männlicher, aggressiver, krimineller Jugendlicher konstruiert wurde, und die heute so in die Jahre gekommen ist, dass sie vermutlich nur noch Wenige amüsant finden (vgl. Bsp. 2).

(2) Quelle: Sprachführer „Kanakisch - Deutsch“ (Eichborn-Verlag) 2003

Es war ma ein krass geile alte Tuss, dem hatte stiefkind. Dem alte Tuss hat immern in sein spiegeln geguckt un den angelabert: "Spiegeln, spiegeln an scheissndreck Wand, wem is dem geilste Tuss in Land?" "Du selbern, isch schwör!" hat dem Spiegeln gesagt. Un weil dem Spiegeln geschwört hat, hat dem dem geglaubt. Abern an eim Tag hat dem scheissndreck spiegeln gesagt, dass dem stieftochthern geilern is. Dem alte Tuss hat eim Typ angelabert un hat gesagt: "Fahr mit dem Arschnloch-Balg in Wald un stesch dem ab, Alder!" Dem Typ hat dem net gemacht, sondern hat dem Balg nur aus Auto geschmeisst. Dann is dem Balg losgelatscht un hat eim susse Haus gesehn un is rein un hat da gepennt. An abend sin dem siebn krasse Swerge gekommen, wo dem Haus gehört un ham gesagt: "Geil, Alder, was fur oberngeile Tuss, kuck ma wie geil dem aussieht." Dem hat am nächstem Morgen dem Tuss gesagt, dass dem da bleiben kann, weil dem obernkrass geil aussieht! Dann sin auf Arbeit gefahrt. Da kam alte Tuss an Haus vorbei un hat dem Balg einem krass genmanipulierte Apfeln gegeben. Dem hat dem gegessen un is tot umgefallt, isch schwör! Als dem Swergen von Arbeit gekommen sin, ham die dem Balg in $3 e r n$ Cabrio geschmeisst un sin Klinik gefahrt. Weil dem Swergen geheizt sin wie Arschlöchern, is dem Balg krass schlecht geworden un hat korreckt auf Ledersitze gekotzt, Alder! Un isch schwör, dem hat wieder gelebt.

Diese Variante des ,Kanakisch“ ist durch ihre humoristische Inszenierung leicht als Konstrukt erkennbar. Ohne jede Basis in der Wirklichkeit der primären ethnischen Merkmale sind hier z.B. die durchgängige Verwendung von dem als Definitartikel, die falschen Partizipialformen (gefahrt) und die Plural- und Kasusmarkierung durch $-n$ in Wörtern wie Spiegeln (Nom.) oder Apfeln, die eher an Formen aus dem Erstspracherwerb erinnern; generalisierte und forcierte primäre ethnische Merkmale sind z.B. die Weglassung des indefiniten Artikels (fur oberngeile Tuss) oder die Weglassung von Präpositionen (sin Klinik gefabrt). 
Die Medienberichterstattung enkodierte aber auch eine andere Sprache, und diese war mit dem Anspruch verbunden, die wirkliche „Kanakensprache" abzubilden. So etwa in der inzwischen berühmten SPIEGEL-Titelgeschichte „Die verlorene Welt“ (14/2006), durch die eine Neuköllner Schule in Berlin, die Rütli-Schule, plötzlich in das Zentrum der öffentlichen Aufmerksamkeit rückte (vgl. Bsp. 3).

(3) (aus: SPIEGEL 14, 2006, Titelgeschichte „Die verlorene Welt")

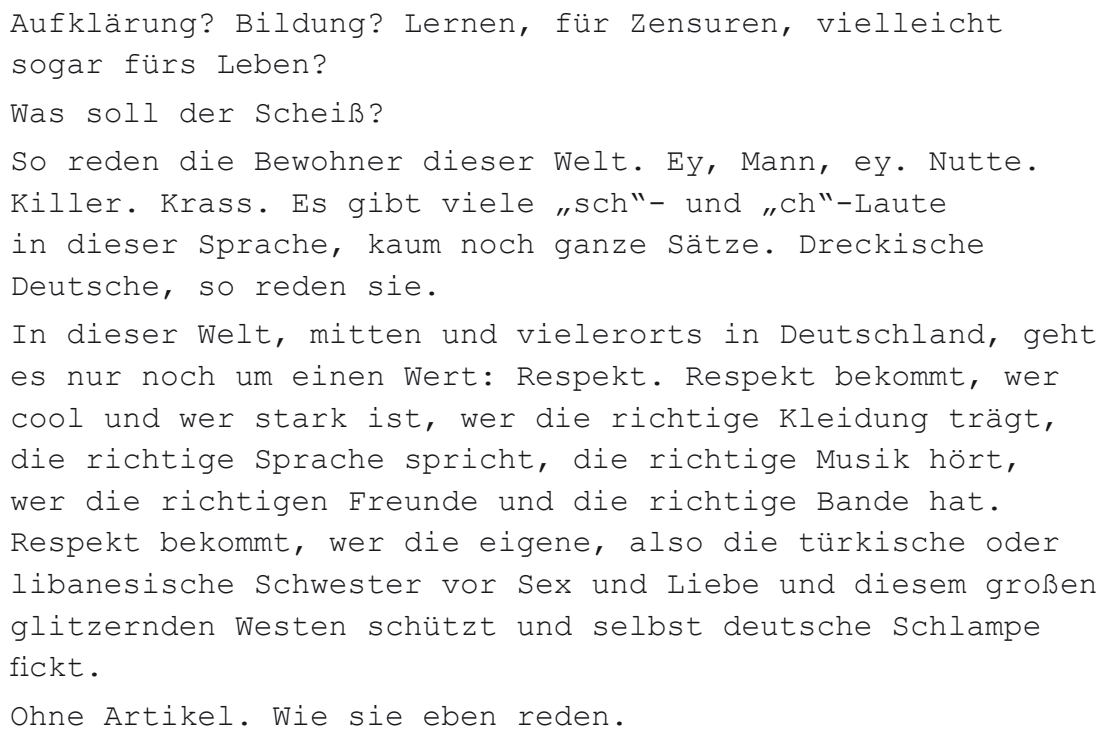

Im Lauf dieses Enkodierungsprozesses bekamen einige sprachliche Merkmale prominenten Status als ethnische Stereotypen, die auch und gerade unter Mitgliedern der Mehrheitsgesellschaft, die keinen oder nur wenig Kontakt mit den Migrationsmilieus hatten, bekannt wurden. Das wichtigste Merkmal auf der Lautebene ist ohne Zweifel die Koronalisierung des palatalen Frikativs in der Silbenkoda - ein bemerkenswerter Prozess der Uminterpretation eines Dialektmerkmals, das in den mitteldeutschen Dialekten weit verbreitet ist. So wurde es möglich, dass im Jahr der Debatte um die Rütli-Schule kurzfristig eine Popgruppe, die Grup Tekkan, bekannt wurde (oder bekannt gemacht wurde), deren einziges rekurrentes ethnisches Erkennungs-Merkmal die $c h$-Koronalisierung war. Die drei Sänger kamen aus Germersheim, also aus dem dialektalen Koronalisierungsgebiet. Dennoch zweifelte niemand daran, dass ihre Ethnizität gerade an der $c h$-Koronalisierung ablesbar war (vermutlich auf dem Hintergrund der Tatsache, dass sie sonst keinerlei dialektale Merkmale auswiesen und sich auch nichtsprachlich in ihrem Stil eindeutig als ethnisch identifizierten). 
(4) (Grup Tekkan, Transkription des Refrains des Songs: „Wo bist du, mein Sonnenlicht?" von 2006)

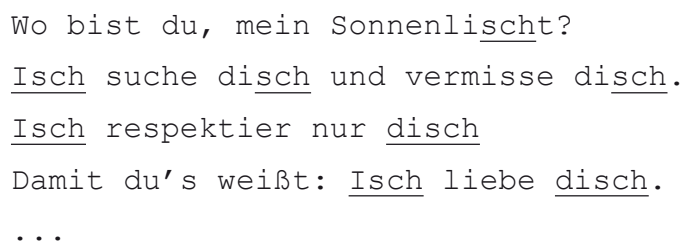

Das zweite sprachliche Merkmal, das (poly-)ethnisch enkodiert wurde, war das Fehlen der Artikel. Beide Merkmale werden in Abschnitt 3 weiter besprochen.

\subsection{Von ethnischen zu polyethnischen Markern}

Ein weiterer Prozess, der in den Mitt-1990er Jahren einsetzte und in den Hamburger Daten aus den letzten Jahren des Jahrtausends (Dirim/Auer 2004) schon deutlich erkennbar ist, ist die Transformation eines vor allem mit Jugendlichen aus türkischen Familien verbundenen (mono-)ethnischen Stils in einen polyethnischen ${ }^{3}$ Stil (Quist 2008). Der Prozess wurde vermutlich durch die mediale Enkodierung befördert, die trotz der Bezeichnung ,Türkendeutsch' in der Regel nicht an einer bestimmten ethnischen Kategorisierung der Sprechertypen interessiert war, sondern über ethnische Gruppen hinweg generalisierte; sicher wurde er aber auch durch die ethnische Diversifizierung der Großstadt-, Ghettos' in den 90er Jahren voran getrieben, die sich aus den großen neuen Flüchtlings- und Asylbewerbergruppen dieser Zeit ergab (Balkankriege, afghanische und iranische Flüchtlinge etc.). Die Indexikalität der sprachlichen Merkmale veränderte sich - statt Türkischsein indizierten sie nun ,Ausländersein', in jenem spezifischen Sinn von ,Ausländer', in dem die Sprecher selbst das Wort verwendeten, also im Sinne von aus dem deutschen mainstream sozial ausgegrenzte Menschen mit nicht-deutschem Familienhintergrund. ${ }^{4}$ Dieser Prozess war bereits um die Jahrtausendwende in den Anfängen zu beobachten; die damals noch klare Dominanz der Türken und Türkinnen belegt allerdings u.a. die Tatsache, dass die von uns in Hamburg untersuchten Jugendlichen nicht-türkischer Herkunft fast nur aus dem Türkischen stammende ${ }^{5}$ Anredeformen und Diskursmarker verwendeten, etwa

Quist (2008) verwendet die Hybridform Multi-Ethnolekt. Der Begriff geht auf Clyne (2000) zurück.

$4 \quad$ Evidenz für diesen polyethnischen Charakter der Merkmale findet sich bei Freywald et al. (2011).

5 Auch wenn diese Wörter etymologisch teils persisch-arabischer Herkunft sind, wurden sie über das Türkische entlehnt. 
(5) lan (>ulan), oğlum, abi (>ağabey), moruk, canım, kıæım, valla(hi), tamam,

kaum solche aus anderen Immigrantensprachen.

Welche ethnischen Gruppen sich dieser polyethnischen Sprechweise anschlossen und welche nicht, ist bis heute nicht empirisch untersucht. Es kann aber wohl als sicher gelten, dass es sich bis heute um einen selektiven Prozess handelt, an dem verschiedene ethnische Gruppen unterschiedlich stark teilnehmen. Wir haben es also nicht mit einer panethnischen, sondern nach wie vor mit einer polyethnischen Sprechweise zu tun.

\subsection{Crossing}

Vor allem durch die Medien vermittelt, finden wir spätestens seit der Jahrtausendwende auch vermehrt die Verwendung stereotypisierter ethnischer Merkmale durch deutsche Jugendliche, die in der Regel nichts mit den Immigrantenmilieus zu tun hatten bzw. sich von ihnen sogar aktiv abgrenzten. Diese Verwendungen sind immer zitathaft und lassen sich am besten unter den von Rampton geprägten Begriff des crossing fassen (Rampton 1995, zusammenfassend Auer 2006). Auch zu diesem Thema gibt es ausführliche Untersuchungen (vgl. z.B. Deppermann 2007).

\subsection{Deethnisierung?}

Crossing muss scharf von dem letzten zu besprechenden Schritt in der Transformation ethnischer Merkmale getrennt werden. Es ist für einige europäische Großstädte behauptet worden, dass sich die polyethnischen Sprechstile von Jugendlichen in den letzten zehn Jahren zu einem „late modern youth style“ de-ethnisiert hätten (vgl. Rampton 2011 und in diesem Band, sowie Cheshire et al. 2011 für London, Madsen 2011 für Kopenhagen, wo der spätmoderne Jugendsprachstil laut ihrer Analyse allerdings vor allem phonetische und lexikalische Merkmale einschließt, kaum die für den deutschen ethnischen Stil diskutierten grammatischen Merkmale). Das würde implizieren, dass die soziale Bedeutungshaftigkeit dieser Sprechweisen als Index für polyethnisches ,Ausländersein'verloren ginge, d.h. auch die Mitglieder der Mehrheitsgesellschaft, die den Stil nicht verwenden, würden ihn nicht mehr mit ,Ausländersein` assoziieren. Der neue Stil werde nicht mehr nur von Jugendlichen mit Migrationshintergrund verwendet, sondern genauso auch von monolingualen Jugendlichen. Ben Rampton spricht in Bezug auf London sogar von einem nicht mehr nur jugendsprachlichen „,contemporary urban vernacular", das sich über das gesamte Altersspektrum hinweg ausbreitet. Systematische Daten für Deutschland fehlen leider. Es gibt aber umgekehrt bisher auch keine Evidenz dafür, dass Erwachsene die Merkmale des polyethnischen Stils beibehalten, die sie als Jugendliche ver- 
wendet haben (oder gar einen solchen Stil erwerben). Keim (2007) zeigt sogar, dass ihre türkischstämmigen Jugendlichen im Übergang zum Erwachsenwerden die ethnischen Merkmale ihrer früheren Sprache ablegen. Dabei spielt in ihrem Fall allerdings nicht nur das Lebensalter eine Rolle, sondern auch die Tatsache, dass sie sich sozial neu positionieren und nicht mehr dem , Ghetto ' zugehörig fühlen. Ob dasselbe auch für junge Erwachsene gilt, die diesen Sprung nicht geschafft haben, ist noch zu untersuchen.

Auch für die Übernahme der ethnischen Sprachmerkmale durch monolingual deutsche Sprecher gab es schon in unseren Hamburger Daten einige Hinweise im Bereich der Morphologie und Syntax (fehlende Artikel und Präpositionen). Wie weit der Prozess fortgeschritten ist, muss empirisch geklärt werden.

\section{Entsteht eine neue Varietät des Deutschen?}

In der bisherigen Darstellung war neutral von ethnischen Sprechweisen, Merkmalen oder Stilen die Rede, Begriffe wie ,Ethnolekt' oder ,Polyethnolekt ${ }^{t}$ wurden vermieden. Ich bin damit der Frage aus dem Weg gegangen, ob die jugendlichen Sprechweisen, mit denen wir hier zu tun haben, Varietätenstatus haben. Sie sind selbstverständlich kein neuer „Dialekt“ (so Wiese 2012) im in der deutschen Linguistik üblichen Sinn des Wortes, ${ }^{6}$ denn sie sind nicht diatopisch definiert, sondern ethnisch oder sozial.

Ob man von Varietäten oder Stilen (Sprechweisen) spricht, ist zunächst eine Frage der Perspektive, d.h. es ist nicht unmöglich, dass dasselbe Set von kookkurrierenden sprachlichen Merkmalen einmal als Teil einer Varietät, das andere Mal als Teil eines sozialen Stils analysiert wird. In der folgenden Gegenüberstellung orientiere ich mich an einem Stilbegriff, der von Eckert und in ihrem Umfeld, der „,third wave sociolinguistics“, entwickelt wurde (Eckert 2004, in Anwendung auf das Thema ethnischer Sprechweisen besonders Quist 2008):

\section{Varietät}

strukturell definiert

bestimmt durch ko-okkurrierende grammatische und ggf. phonologische Merkmale

\section{Stil}

ethnografisch definiert

bestimmt durch linguistische und andere semiotische Merkmale, die soziale Bedeutung haben/bekommen

\footnotetext{
In der britischen Soziolinguistik wird dialect oft synonym mit variety gebraucht, etwa wenn vom standard dialect die Rede ist. In der kontinentaleuropäischen Tradition sind Dialekte immer diatopisch gebundene Varietäten. Es scheint mir keinen Grund zu geben, diese etablierte Definition aufzugeben, zumal mit ,Varietät ${ }^{\dagger}$ ja ein neutraler Begriff zur Verfügung steht.
} 


\section{Varietät}

etisch: definiert aus der Perspektive des Wissenschaftlers

die Merkmale werden als System verstanden und analysiert

stabil, feste Grenzen

Verletzungen von Kookkurrenzrestriktionen führen zu inakzeptablen Äußerungen

bezogen auf eine Sprachgemeinschaft

\section{Stil}

emisch: definiert aus der Perspektive der sozial Handelnden

die Merkmale werden als Form der sozialen Praxis verstanden und analysiert

kontextabhängig, prozessual, fließend, schnell neu konfigurierbar

Verletzungen von Kookkurrenzrestriktionen führen zu neuen/ anderen sozialen Bedeutungen (oder machen den Sprecher unauthentisch)

bezogen auf communities of practice (Wenger 1998), d.h. auf eine kleine Gruppe

Abb. 3: Stile und Varietäten im Kontrast

Stile werden nach Eckert kleinräumig in sozialen Gruppen - so genannten communities of practice (Wenger 1998) - entwickelt und unterliegen schnellen Veränderungen. Sie können schnell rekonfiguriert werden, und diese Rekonfigurationen werden von den Teilnehmern genutzt, um sich selbst in einem sozialen Raum zu verorten, in dem sie ihre eigene soziale Identität in Opposition zu relevanten anderen Milieus konstruieren. Die Konstruktion sozialer Identitäten ist daher ,a process of bricolage - an appropriation of local and extra-local linguistic resources in the production not just of a pre-existing persona but of new twists on an old persona" (Eckert 2000, S. 214). Nimmt man noch die Interaktion zwischen medialen und individuellen Identitätskonstruktionen hinzu, die Eckert nicht zentral berücksichtigt, die aber im Falle ethnischer Identitätskonstruktionen durch sprachliche Stilmerkmale eine wesentliche Rolle spielt, kommt diese Beschreibung den sozialen Praktiken in den Immigrantenmilieus der deutschen Großstadtvierteln recht nahe. Der Eckertsche Stilbegriff betont die Gebundenheit der den Stil konstituierenden semiotischen Merkmale an kleine Gruppen direkt miteinander kommunizierender Sprecher und Sprecherinnen, die diese semiotischen Ressourcen aus einem Pool schöpfen, der selbst durch Varietäten bestimmt sein kann. Diese kleinräumige, selektive Aneignung von Bruchstücken aus Varietäten ist auch in polyethnischen Milieus zu beobachten, sie steht aber in einem Spannungsverhältnis zur großräumigen, v.a. medialen Enkodierung stilistischer Muster. 
Die Relevanz sprachlicher Merkmale für soziale Stile schließt also nicht aus, dass dieselben Merkmale auch als Elemente von sprachlichen Varietäten betrachtet werden können, die aus der Perspektive des Linguisten definiert werden und durch systemhaft miteinander ko-okkurrierende Merkmale bestimmt sind. So argumentiert Wiese (2012), dass das ,Kiezdeutsch“ ein „eigenes System“ bilde - vergleichbar mit anderen Varietäten des Deutschen (2012, S. 105). In der Tat ist es wichtig zu unterstreichen, dass das ,Kiezdeutsch“ keine Ansammlung von individuellen Fehlern ausländischer Sprecher ist, die des Deutschen nicht mächtig sind. Es handelt sich bei den ethnischen Merkmalen meist (siehe unten) um systematische Abweichungen von der Standardvarietät des Deutschen. Allerdings ergibt sich daraus noch nicht automatisch ihr Varietätenstatus, denn natürlich weiß man in der Soziolinguistik seit vielen Jahrzehnten, dass auch Variation innerhalb einer Varietät systematisch organisiert ist. Um tatsächlich von sprachlichen Varietäten sprechen zu können, müssen zumindest einige der folgenden Merkmale vorliegen:

- die Jugendlichen sollten die die Varietät definierenden sprachlichen Merkmale mit einer gewissen Häufigkeit verwenden;

- die einzelnen Merkmale sollten nicht in Isolation, sondern in Kookkurrenz miteinander auftreten;

- die Merkmale sollten wesentliche Eigenschaften des sprachlichen Systems betreffen, insbesondere nicht nur den Wortschatz oder die Lautstruktur, sondern auch die Morphologie und Syntax.

In diesem Abschnitt sollen nun einige der sprachlichen Merkmale in Bezug auf die genannten Parameter diskutiert werden. Grundlage sind Daten, die 2010-2012 in Jugendzentren und Schulen in so genannten Brennpunktvierteln der Stadt Stuttgart von Daniela Picco und Vanessa Siegel im Rahmen ihrer laufenden Promotionsprojekte aufgenommen wurden. Im Augenblick werden ca. 20 Sprecher und Sprecherinnen analysiert, die 14-19 Jahre alt sind. Es handelt sich mehrheitlich um Jugendliche mit türkischem bzw. türkisch-kurdischem Familienhintergrund, aber auch solche mit Eltern aus dem Kosovo, aus Bulgarien, Italien, Tunesien gehören zur Informantengruppe. ${ }^{7}$ Im vorliegenden Beitrag werden lediglich Daten von zehn Sprechern berücksichtigt.

\subsection{Lautliche Eigenschaften}

Die Phonetik und Phonologie ethnischer Sprechweisen ist trotz vieler charakteristischer Merkmale noch kaum systematisch untersucht. Das stereotypi-

Die Auswertung ist noch nicht abgeschlossen. 
sierte Merkmal der $c h$-Koronalisierung (/ç/ > [६]) scheint in der Wahrnehmung alle anderen lautlichen Eigenschaften in den Hintergrund gedrängt zu haben. Die erste Frage an unser Material war daher, wie häufig die $c h-$ Koronalisierung überhaupt auftritt. Dazu wurden alle Tokens für die Wörter ich und mich sowie, falls diese keine 100 Beispiele ergaben, außerdem die Realisierungen von sich, dich und nich $(t)$ ausgezählt (bis die Daten erschöpft oder ca. 100 Belege gesammelt waren). Die Ergebnisse finden sich in Tabelle 1. Die Koronalisierung kann unterschiedlich stark sein, denn ihre artikulatorische Grundlage (Kontakt zwischen Palatum und Zunge breiter und oft auch mehr nach hinten verlagert) ist graduell. Entsprechend werden in der Auszählung klar koronalisierte Realisierungen von partieller Koronalisierung unterschieden. Im Kontext vor Verben, die mit einem alveo-palatalen Frikativ beginnen (also etwa in ich schwör), ist die Realisierung von /ç/ als [c] oder sogar [S] kein Ergebnis der Koronalisierung (allein), sondern (vor allem) der Assimilation an den Anlaut der folgenden Silbe. Diese Fälle wurden daher nicht berücksichtigt. Die Werte in Tabelle 1 beziehen sich also lediglich auf die prozentualen Anteile der (partiellen) Koronalisierung in nicht-assimilierenden Kontexten.

\begin{tabular}{|l|c|c|c|c|c|c|}
\hline & $\begin{array}{c}\text { Herkunftsland } \\
\text { Familie }\end{array}$ & Alter & $\begin{array}{c}\text { vollständige oder } \\
\text { weitgehende } \\
\text { Koronalisierung }\end{array}$ & $\begin{array}{c}\text { partielle } \\
\text { Korona- } \\
\text { lisierung }\end{array}$ & $\begin{array}{c}\text { palataler } \\
\text { Frikativ }\end{array}$ & n= \\
\hline MA N_JH_1,6 & Portugal & 18 & $11 \%$ & $7 \%$ & $82 \%$ & 94 \\
\hline FU N_JH_1 & Türkei & 15 & $1 \%$ & $15 \%$ & $84 \%$ & 85 \\
\hline FAB N_JH_2 & Italien & 16 & $2 \%$ & $9 \%$ & $89 \%$ & 64 \\
\hline MA2 N_JH_2, & Italien & 15 & $3 \%$ & $5 \%$ & $92 \%$ & 101 \\
\hline NORS_3 N_JH_5 & Türkei & 15 & $9 \%$ & $14 \%$ & $77 \%$ & 112 \\
\hline BU N_JH_5 & Türkei & 15 & $9 \%$ & $14 \%$ & $77 \%$ & 100 \\
\hline LO O_JH_1 & Bosnien & 15 & $2 \%$ & $3 \%$ & $95 \%$ & 59 \\
\hline ES N_RS_1 & Kosovo & 17 & $18 \%$ & $36 \%$ & $46 \%$ & 100 \\
\hline $\begin{array}{l}\text { ER BC_JA_08, } \\
\text { 09 }\end{array}$ & Türkei & 14 & $2 \%$ & $6 \%$ & $92 \%$ & 100 \\
\hline MV BC_JA_5 & Türkei & 15 & $2 \%$ & $4 \%$ & $93 \%$ & 92 \\
\hline
\end{tabular}

Tab. 1: Koronalisierung in ich, mich (dich, sich, nich $(t))$ in nicht-assimilierenden Kontexten bei zehn männlichen Jugendlichen mit unterschiedlichem sprachlichen Hintergrund 


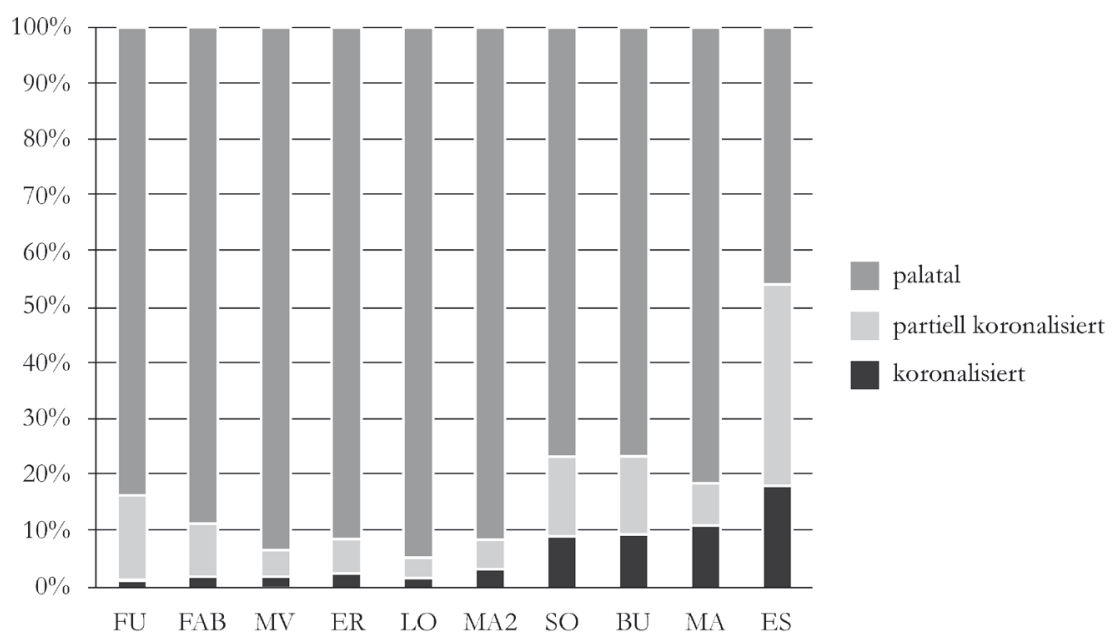

Abb. 4: Prozentualer Anteil der Koronalisierung in ich, mich (dich, sich, nich $(t))$ in nicht-assimilierenden Kontexten bei zehn männlichen Jugendlichen mit unterschiedlichem sprachlichen Hintergrund

Aus durchschnittlich nur knapp 6\% voll koronalisierten Formen, 83\% palatalen Realisierungen und 11\% Zwischenformen lässt sich ableiten, dass dieses Stereotyp ethnolektaler Sprechweise zumindest bei Stuttgarter Jugendlichen mit Migrationshintergrund keine wesentliche Rolle spielt (vgl. Abb. 4). Das Merkmal ist bei sechs der Jugendlichen praktisch nicht existent, und lediglich ein Informant (der Kosovo-Albaner ES) verwendet es regelmäßig, wenn auch in Alternanz mit der palatalen Form; in seinem Fall sind die teils oder voll koronalisierten Varianten etwa genauso häufig wie die palatalen Realisierungen.

Allerdings entspricht die nicht-koronalisierte Realisierung des auslautenden Frikativs nach vorderem Vokal keineswegs immer der standardsprachlichen bzw. aus den autochthonen Nicht-Standard-Varietäten bekannten Lautung. So lassen sich im Material insbesondere zahlreiche Fälle von Verstimmhaftung des auslautenden Frikativs beobachten. Bemerkenswert ist außerdem, dass die nicht-koronalisierte Realisierung auch in vielen Äußerungen vorkommt, die aufgrund ihrer sonstigen Phonetik eindeutig ethnisch markiert sind. Das phonetisch-phonologische System der Jugendlichen ist insgesamt klar durch eine Reihe von nicht-autochthonen Merkmalen gekennzeichnet, die sowohl segmentaler als auch suprasegmentaler (prosodischer) Art sind. In den Daten gehören dazu u.a. 
- die starke Stimmhaftigkeit der im autochthonen, vor allem südwestdeutschen Deutsch schwach oder gar nicht stimmhaften, als Lenes realisierten Plosive /b, d, g/ sowie der Frikative /s, $\int, j /$ sowie / $/ \mathrm{j}$ in wortinitialer und -medialer, aber auch in Auslautposition;

- im Vergleich zum autochthonen Deutsch die artikulatorische Fortisierung des / $\mathrm{r}$-/Lautes in silbeninitialer Position, und zwar entweder als apikaler r-Laut oder - häufiger - durch eine starke frikativische Geräuschbildung, die für den deutschen Südwesten völlig untypisch ist;

- die tendenzielle Monophthongierung der Diphthonge durch Angleichung von on- und offglide. Die Verkürzung der Distanz zwischen Anund Abglitt ist auch für das Schwäbische typisch; während sie dort jedoch durch Hebung des Anglitts erreicht wird (der schwa-ähnliche Qualität bekommt), scheint sie im ethnischen Stil eher durch die Senkung des offglides erreicht zu werden; eine Zentralisierung des Anglitts ist nicht zu beobachten;

- die teilweise gespannte Realisierung der im autochthonen Deutschen kurzen Vokale /u, o/; umgekehrt scheint der vordere Hochvokal (/i/) oft stark zentralisiert zu werden.

Diese segmentalen Merkmale werden (mit Ausnahme der r-Varianten) nicht kategorisch realisiert, sondern variieren sehr stark. Sie sind aber ohne Frage so rekurrent und frequent, dass praktisch alle Äußerungen der Sprecher als nicht-autochthon erkennbar sind. Dazu kommen prosodische Merkmale. Die Tendenz zur Rhythmisierung durch starke Hervorhebung von pränuklearen (Nebenakzent-)Silben, die Kern (2011) für Berlin feststellt, ist jedoch ein relativ seltenes Kontextualisierungsmittel, das nur für bestimmte Äußerungstypen eingesetzt wird. Wichtiger für die Prosodie der untersuchten Sprecher scheint ein spezifisches Zusammenspiel zwischen der Tendenz zur extremen Reduktion unbetonter Silben, vor allem im Bereich vor der Nukleussilbe in der Intonationsphrase einerseits, und einer Tendenz nur Nicht-Reduzierung von Silben, die im autochthonen Deutsch zu Schwa reduziert werden, andererseits.

Die Koronalisierung spielt im Gefüge dieser Merkmale jedenfalls keine wichtige Rolle. Dies erinnert an das vollständige Fehlen der Koronalisierung in den Hamburger Daten aus der Zeit um 2000 (Dirim/Auer 2004); die soziale Bedeutung der Koronalisierung ist jedoch in Stuttgart eine andere. Während die Koronalisierung in Hamburg keine soziale Bedeutung hatte, wird sie von den Stuttgarter Sprechern aktiv vermieden. Dies lässt sich im folgenden Gesprächsausschnitt erkennen, in dem ein Sprecher den anderen explizit wegen seiner $c h$-Koronalisierung kritisiert: 
(6) (BC_JA_01, 426-432)

(IL ist ein 15-jähriger Kosovo-Albaner, MV sein 15-jähriger türkischer Freund; es geht um die Schulprojekte der Jugendlichen, nach denen JS, die Interviewerin EX, gefragt hat)

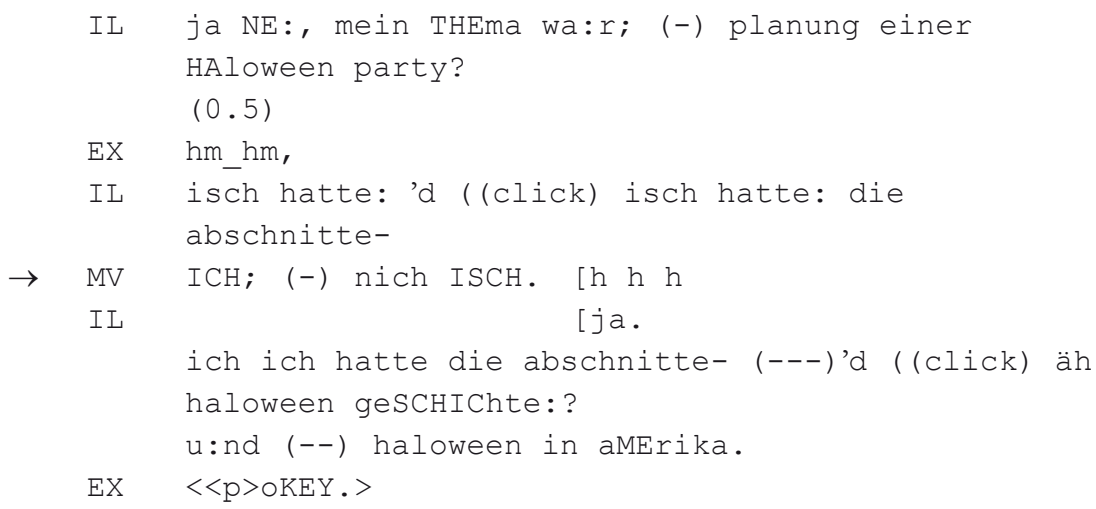

Das für (sekundäre?) ethnische Stile enkodierte Merkmal der Koronalisierung ist den Jugendlichen also bewusst und wird - jedenfalls in einem Gespräch mit einer jungen Erwachsenen - als unpassend kritisiert. (Man beachte, dass MV in seiner eigenen Sprachproduktion während der Aufnahme nur in 2\% der Fälle koronalisiert; vgl. Tab. 1.) Wir haben es mit einer bewussten Abwehr allzu ,kanaksprachlich' konnotierter Sprechweisen zu tun, die evtl. auch durch die mediale Konstruktion des Stereotyps bedingt ist. $\mathrm{Da}$,Kanaksprak ${ }^{6}$ als typisch für das Berliner Milieu enkodiert ist, passt dazu auch, dass für unsere Informanten gerade Jugendliche aus Berlin Neukölln (die sie übrigens nicht über die Print- oder Funkmedien kennen, sondern aus einer Chatgroup) eine relevante Gegengruppe darstellen, von der sie sich explizit abgrenzen:

(7) (BC_JA_02)

(aus demselben Gespräch; Fisch = allgemeine abwertende Bezeichnung)

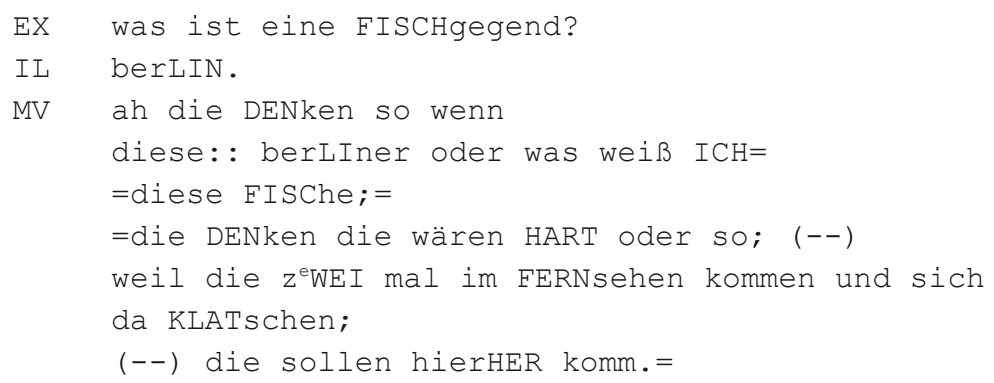




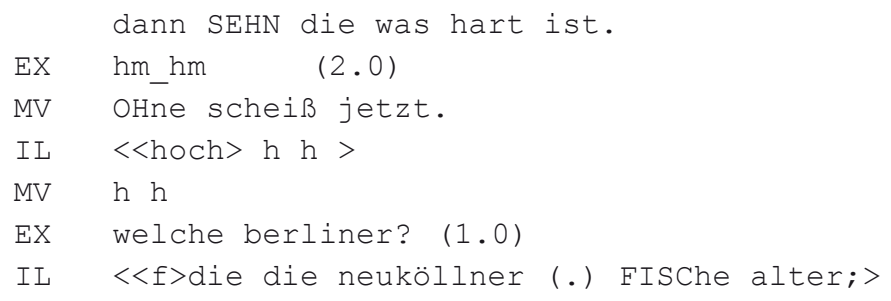

Der Verweis auf die Auftritte der Neuköllner Jugendlichen im Fernsehen spielt auf die mediale Inszenierung der polyethnischen Berliner ,Szene' an. Es ist also zumindest wahrscheinlich, dass die Koronalisierung mit der enkodierten ,Ghettovarietät ${ }^{`}$ des Berliner ,Kiez' in Verbindung gebracht und gerade deshalb abgelehnt wird.

Auffallend ist in diesem Zusammenhang der Betonung regionaler (Stuttgarter) Identitäten, dass die Phonetik und Phonologie der Sprecher und Sprecherinnen kaum dialektale Merkmale des Schwäbischen aufweist (auch wenn die pro-Stuttgarter Parteinahme im Ausschnitt (7) dies vielleicht erwarten ließe). Neben seltenen allgemein-süddeutschen Merkmalen wie net für nicht scheint sich der Einfluss der regionalen Umgebungssprache auf gelegentliche Senkungen von std. /e:/ (wie in [lв:ьә] Lehre) und auf die gelegentliche s-Palatalisierung in der 2. Person des Flexionsparadigmas zu beschränken, diese teils mit ironischem, zitathaftem Charakter. Ausschnitt (8) ist eines der wenigen Beispiele in unseren Daten, in denen die Palatalisierung verdichtet auftritt. Der Erzähler (ein 19-jähriger Kosovo-Albaner) erzählt von einer Auseinandersetzung mit seiner deutschen Freundin, die er hier direkt adressiert. Das Suffix /-S/ für die 2.Ps.Sg. ist hier offenbar ein Kontextualisierungsverfahren, das den deutschen Kontext der reportierten Situation unterstreicht:

(8) (N_JH_3)

(LE konfrontiert seine deutsche Freundin damit, dass er sie mit anderen Männern beobachtet hat)

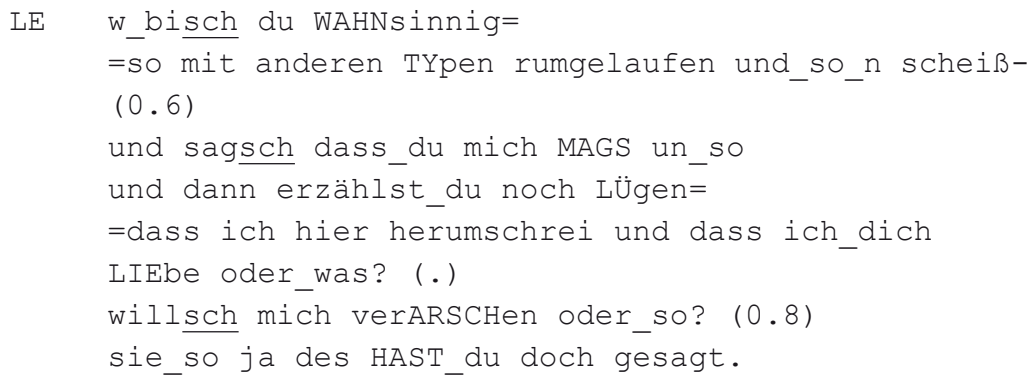


Neben drei palatalisierten Versionen des Suffixes verwendet der Sprecher allerdings auch drei standardsprachliche. Außer der Palatalisierung kommen im Ausschnitt keine anderen schwäbischen Merkmale vor.

Im Gesamtkorpus sind knapp 10\% der Realisierungen von du bist palatalisiert $(\mathrm{n}=239)$, allerdings lediglich $2 \%$ aller Vorkommen von es ist ( $n=2929$ ). Möglicherweise tendiert die phonologische Regel der s-Palatalisierung vor Obstruent (die morphologisch eingeschränkt und daher relativ komplex ist: Zwischen den beiden Obstruenten darf keine morphologische Grenze stehen; vgl. er /ist/ vs. $d u$ /ift/ > essen) zur Morphologisierung und wird vor allem im Suffix der 2.Ps.Sg. verwendet.

Im Großen und Ganzen ist die Sprechweise unserer Jugendlichen aber erstaunlich wenig regional markiert; sie weist sogar orthoepische phonetische Züge auf, die im oberdeutschen Sprachraum auch in der Standardvarietät selten sind. Etwa wird silbenanlautendes /s/ sehr oft stimmhaft realisiert, und auslautende -ig-Endungen oft mit palatalem Frikativ.

Der Blick auf das Lautsystem unserer Sprecher lässt also erkennen, dass systemhaft bestimmte Eigenschaften auftreten. Es ist daher möglich und gerechtfertigt, von einer ethnolektalen Lautstruktur zu sprechen. Die Koronalisierung gehört in Stuttgart allerdings höchstens am Rande dazu.

\subsection{Grammatische Merkmale}

Auch einige morpho-syntaktische Konstruktionen sind unter dem Einfluss der Medien als ,Kiezdeutsch' kodiert worden, so light verb-Konstruktionen wie das eingangs zitierte ich mach dich Messer oder ich mach dich Krankenhaus. Allerdings ist die Beleglage hier dünn, und die Stuttgarter Daten lassen bisher keine Aussage darüber zu, ob solche Konstruktionen systematisch vorkommen. (Wie schon erwähnt, ist zumindest das erste Beispiel wohl eine mediale Erfindung.) Bei anderen angeblichen Merkmalen des ,Kiezdeutsch zeigt sich schnell, dass sie im informellen, gesprochenen autochthonen Deutsch genauso vorkommen wie bei Jugendlichen mit Migrationshintergrund. Das trifft sicherlich auf die Verwendung von so als Fokusmarker zu. Wiese sieht dieses Merkmal als Beleg für den kreativen und innovativen Charakter des ,Kiezdeutsch':

Ein weiteres charakteristisches Merkmal von Kiezdeutsch ist die Verwendung von „so“ an Stellen, an denen man es im Standarddeutschen nicht erwarten würde. Hier zur Verdeutlichung noch einmal zwei der Beispielsätze aus der Einleitung: „Ich höre Alpa Gun, weil der so aus Schöneberg kommt.“ / „Ich hab meiner Mutter so Zunge rausgestreckt, so aus Spaß.“

Im Standarddeutschen hat die Partikel „so“ mehrere Funktionen, insbesondere kann sie Vergleichsrelationen ausdrücken („So schnell wie Anja“) und Intensität markieren (,So hoch!“). In der gesprochenen Sprache wird „so“ darüber hinaus als sogenannter Quotativmarker verwendet, zur Einleitung von Zitaten („Ich 
dann so: ,Was ist denn hier los?“"). Gemeinsam ist diesen Funktionen, dass „so“ einen Bedeutungsbeitrag leistet, der auf „Wie?“ antwortet und umschrieben werden kann mit ,auf diese Art“.

In Kiezdeutsch kommt noch eine neue Funktion hinzu, bei der dieser Bedeutungsbeitrag dann entfällt: In den Beispielen steht „so“ jeweils vor dem so genannten Fokus des Satzes, jenem Teil, der die neue, besonders hervorzuhebende Information liefert. Im ersten Beispiel ist dies „aus Schöneberg“: Diese Ortsangabe liefert die besonders hervorgehobene Information, sie gibt den Grund an, aus dem die Sprecherin den Rap-Sänger Alpa Gun besonders mag. Im zweiten Beispiel ist die wichtige Information im ersten Teil die Handlung „Zunge rausgestreckt“, im zweiten Teil die Information, dass dies ,aus Spaß“ geschah. In allen Fällen markiert „so“ den jeweiligen Fokusausdruck. (Wiese 2010, S. 37)

Ein Blick in beliebige neuere Korpora des mündlichen, autochthonen Deutsch belegt aber, dass es sich bei diesem so um ein allgemeines Merkmal der gesprochenen Umgangssprache handelt, und dass die im obigen Zitat gegebene Beschreibung des gesprochenen Standarddeutschen unvollständig ist. ${ }^{8}$ Dazu die folgenden Belege:

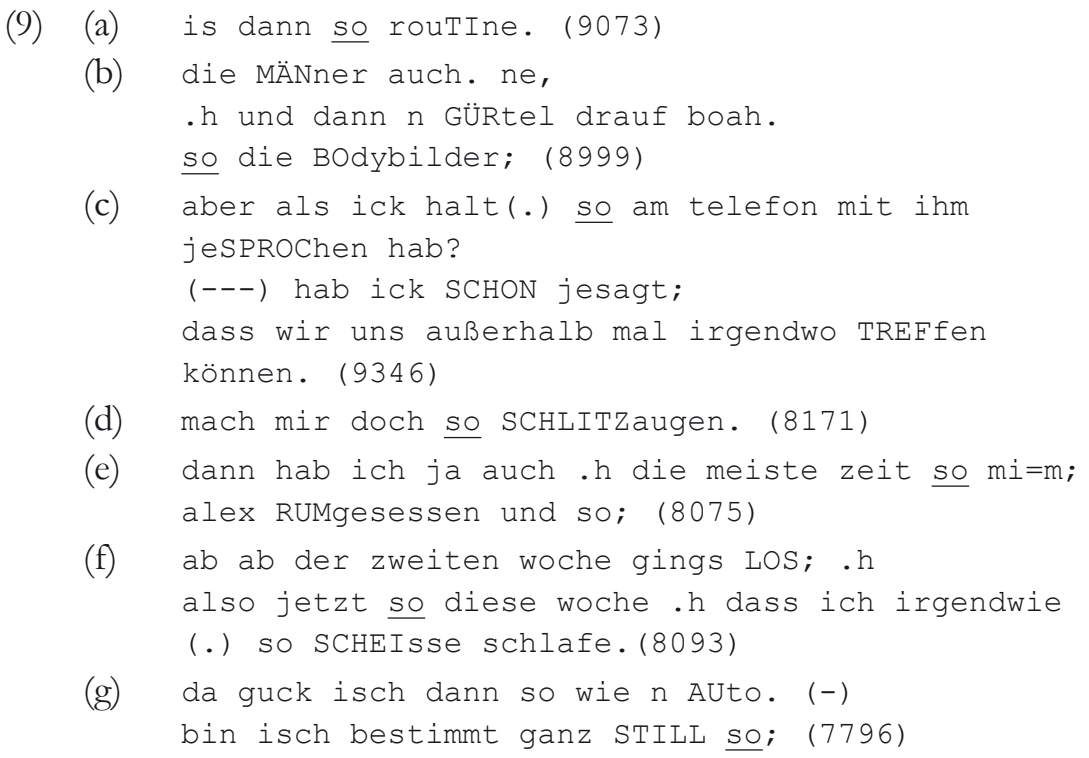

All diese Beispiele stammen von erwachsenen, deutsch-monolingualen Sprechern, die auch sonst keinerlei ethnischen Marker verwenden. ${ }^{9}$ Wenn sich

$8 \quad$ In Wiese (2011) und (2012) (in letzterem Buch erscheint der Satz Die gucket so zu dir so noch als Beispiel für ,Kiezdeutsch' auf dem Cover) wird diese Position allerdings revidiert und konzediert, dass so als „Fokusmarker“ auch außerhalb des Kiezdeutsch vorkomme. Das wird als Evidenz dafür gewertet, dass dieser „,neue Dialekt“ eng ins System des Deutschen eingebunden ist. Tatsächlich ist diese Verwendung von so einfach nicht kiezdeutsch.

9 Hier: aus der 1. Staffel der Reality-TV-Produktion „Big Brother“. 
also im Deutschen mit so ein neuer Fokus-Marker ${ }^{10}$ entwickelt hat, so ist diese Entwicklung sicherlich nicht typisch für eine neu entstehende Varietät des Deutschen. Es handelt sich vielmehr um ein allgemein verbreitetes Merkmal der gesprochenen deutschen Sprache. Dieses Merkmal kommt selbstverständlich auch in den Daten unserer Stuttgarter Jugendlichen vor; hier einige Beispiele:

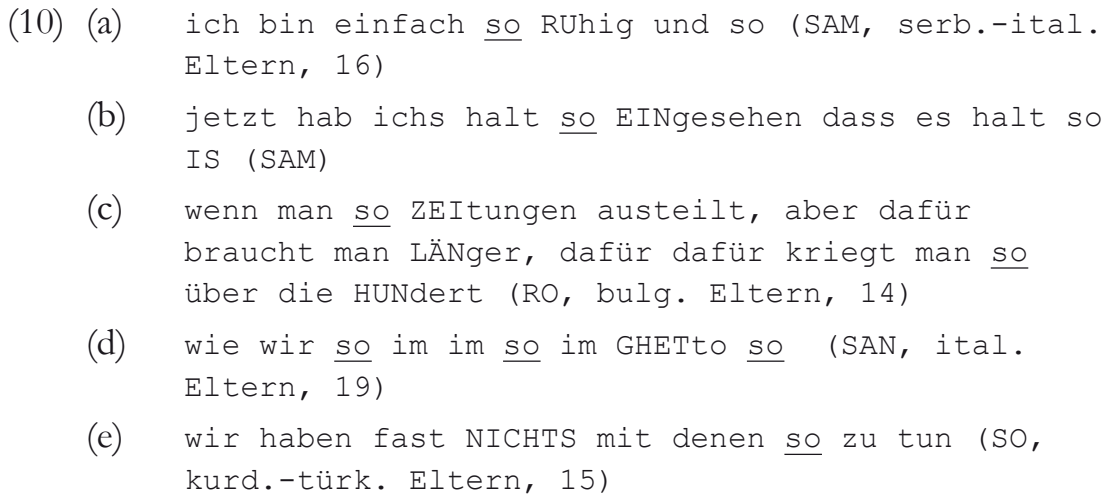

Der Fokusmarker so ist also kein typisches Merkmal des ,Kiezdeutsch‘. Es lassen sich in der Sprache unserer Informanten allerdings zahlreiche andere grammatische Merkmale finden, die deutlich von der Struktur des autochthonen Deutschen abweichen. Auch hier können erste Ergebnisse der Stuttgarter Untersuchung ${ }^{11}$ einen vorläufigen Einblick geben (vgl. Tab. 2).

\begin{tabular}{|c|c|c|c|c|c|c|c|c|c|c|c|c|c|}
\hline $\begin{array}{l}\text { Spre- } \\
\text { cher }\end{array}$ & $\begin{array}{c}\text { Auf- } \\
\text { nahmen }\end{array}$ & $\begin{array}{l}\text { kein } \\
\text { indef. } \\
\text { Art. }\end{array}$ & $\begin{array}{l}\text { indef. } \\
\text { Art. }\end{array}$ & $n=$ & $\begin{array}{l}\text { Nicht- } \\
\text { Reali- } \\
\text { sierung } \\
\%\end{array}$ & $\begin{array}{l}\text { kein } \\
\text { def. } \\
\text { Art. }\end{array}$ & $\begin{array}{l}\text { def. } \\
\text { Art. }\end{array}$ & $n=$ & $\begin{array}{l}\text { Nicht- } \\
\text { Reali- } \\
\text { sierung } \\
\%\end{array}$ & $\begin{array}{l}\text { keine } \\
\text { Präp }\end{array}$ & $\begin{array}{l}\text { Präp } \\
\text { (\& } \\
\text { Art) }\end{array}$ & $n=$ & $\begin{array}{l}\text { Nicht- } \\
\text { Reali- } \\
\text { sierung } \\
\%\end{array}$ \\
\hline MV & BC_JA_01 & 15 & 14 & 29 & 51,7 & 7 & 42 & 49 & 14,2 & 4 & 70 & 74 & 5,4 \\
\hline ER & BC_JA_08 & 4 & 27 & 31 & 12,9 & 12 & 23 & 35 & 34,2 & 7 & 43 & 50 & 14,0 \\
\hline LO & O_JH_1 & 3 & 14 & 17 & 17,6 & 4 & 18 & 22 & 18,1 & 20 & 48 & 68 & 29,4 \\
\hline $\mathrm{FU}$ & N_JH_1 & 5 & 24 & 29 & 17,2 & 3 & 26 & 29 & 10,3 & 2 & 56 & 58 & 3,4 \\
\hline MA & N_JH_1 & 6 & 23 & 29 & 20,7 & 7 & 40 & 47 & 14,8 & 1 & 78 & 79 & 1,3 \\
\hline
\end{tabular}

$\overline{10} \mathrm{Ob}$ es sich tatsächlich um einen Fokusmarker handelt, sei dahin gestellt. Beispiele wie (9d) weisen wohl noch auf eine frühere Verwendung als Vagheitsmarker (bedge) hin; vgl. Auer (1981). Im heutigen gesprochenen Deutsch ist diese Verwendungsweise allerdings nicht mehr dominant. Andererseits sind Beispiele wie (10e) auch nur schwer mit der Interpretation als Fokusmarker kompatibel, denn zu tun ist hier sicher nicht der Satzfokus.

11 Die Auswertung in Tabelle 2 ist Teil des laufenden Promotionsprojekts von Vanessa Siegel. Ich danke ihr für die Überlassung von Daten und Auswertungen. 


\begin{tabular}{|c|c|c|c|c|c|c|c|c|c|c|c|c|c|}
\hline $\begin{array}{l}\text { Spre- } \\
\text { cher }\end{array}$ & $\begin{array}{c}\text { Auf- } \\
\text { nahmen }\end{array}$ & $\begin{array}{l}\text { kein } \\
\text { indef. } \\
\text { Art. }\end{array}$ & $\begin{array}{l}\text { indef. } \\
\text { Art. }\end{array}$ & $n=$ & $\begin{array}{l}\text { Nicht- } \\
\text { Reali- } \\
\text { sierung } \\
\%\end{array}$ & $\begin{array}{l}\text { kein } \\
\text { def. } \\
\text { Art. }\end{array}$ & $\begin{array}{l}\text { def. } \\
\text { Art. }\end{array}$ & $n=$ & $\begin{array}{l}\text { Nicht- } \\
\text { Reali- } \\
\text { sierung } \\
\%\end{array}$ & $\begin{array}{l}\text { keine } \\
\text { Präp }\end{array}$ & $\begin{array}{l}\text { Präp } \\
(\& \\
\text { Art) }\end{array}$ & $n=$ & $\begin{array}{l}\text { Nicht- } \\
\text { Reali- } \\
\text { sierung } \\
\%\end{array}$ \\
\hline ES & N_RS_1 & 5 & 48 & 53 & 9,4 & 6 & 37 & 43 & 13,9 & 5 & 79 & 84 & 6,0 \\
\hline BU & N_JH_5 & 3 & 20 & 23 & 13,0 & 7 & 34 & 41 & 17,0 & 8 & 68 & 76 & 10,5 \\
\hline $\mathrm{SO}$ & N_JH_5 & 3 & 22 & 25 & 12,0 & 6 & 33 & 39 & 15,3 & 5 & 71 & 76 & 6,6 \\
\hline FAB & N_JH_2 & 6 & 44 & 50 & 12,0 & 5 & 34 & 39 & 12,8 & 10 & 70 & 80 & 12,5 \\
\hline MA2 & N_JH_2 & 3 & 18 & 21 & 14,3 & 5 & 42 & 47 & 10,5 & 5 & 60 & 65 & 7,7 \\
\hline
\end{tabular}

Tab. 2: Realisierung von definitem und indefinitem Artikel sowie Realisierung der Präposition in Präpositionalphrasen bei zehn männlichen Jugendlichen mit unterschiedlichem sprachlichen Hintergrund

\subsubsection{Nicht-Verwendung der Präposition}

In der untersuchten Aufnahme der zehn Jugendlichen, die bereits als Grundlage der phonologischen Untersuchung (Tab. 1) dienten, kommen 710 Phrasen vor, die im autochthonen (Standard- oder Nicht-Standard-)Deutschen als Präpositionalphrasen realisiert werden müssten. In 67 Beispielen, also 9,4\%, fehlt die Präposition. Durchschnittlich verwendet ein Sprecher in 9,1\% aller Fälle keine Präposition.

Das ist ein relativ kleiner Prozentsatz - es ist also keineswegs der Fall, dass die Sprecher eine Varietät des Deutschen sprechen würden, in der grundsätzlich die Präpositionen fehlen, wie manchmal verkürzt gesagt wird. (Die prozentualen Anteile der standardkonformen Realisierungen von PPs variierten zwischen den Sprechern von ca. 70\% bis ca. 100\%.) Die Zahlen über alle PPs hinweg verschleiern allerdings, dass es grammatische Kontexte gibt, in denen die präpositionslose Ausdrucksweise in der Sprache der Jugendlichen möglich ist, während sie in anderen unmöglich oder unwahrscheinlich ist. Die wichtigste Einschränkung ist, dass die Präpositionen fast nur in Ortsangaben fehlen können. (Zu den wenigen Ausnahmen in unserem Material gehören Verkürzungen von festen Wendungen wie zum Beispiel, das ein Sprecher als Beispiel realisiert.) Aber auch räumliche Beziehungen können keineswegs alle ohne Präpositionen ausgedrückt werden. Vielmehr ist diese Möglichkeit auf lokale (Lokalisierung innerhalb eines Raums oder einer Institution) und direktionale Angaben (Richtung einer Bewegung) beschränkt. Im untersuchten Material gibt es bisher keine Evidenz, dass andere Raumangaben, die etwa den Ursprung einer Bewegung, eine Pfad-Angabe oder die Nähe zu einem Referenzpunkt ausdrücken, ohne Präposition realisiert werden könnten. Hier wird die Präposition durchweg realisiert, vgl.: 
(11) (a) da bei Stuttgart Ost (LO O_JH_1 1050)

(b) ist da bei Georgiengrenze da (ER BC_JA_08 188)

(c) durch Bulgarien und so (ER BC_JA_08 145)

(d) aus welchem Land kommen Sie? (ER BC_JA_08 728)

(e) wir kommen aus stuttgart (MV BC_JA_02 115)

(f) der Lukas von da drüben (BU N_JH_5 719)

(g) ich steh noch vor der Tür (MA2 N_JH_2 903)

(h) in der Nähe von Porto (MA N_JH_1 544)

(i) da war $n$ stuhl neben mir (MA N_JH_1 898)

(j) die fliegen so über mein Dach (SO N_JH_5 1044)

Ebenso ist es nicht möglich, eine pronominale PP ohne Präposition zu realisieren:

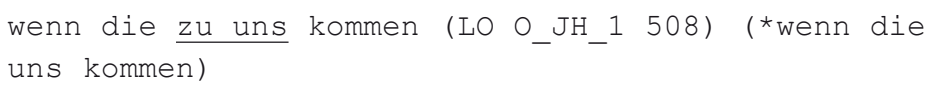

Schließlich konnten wir nie beobachten, dass Präpositionen in festen Wendungen und in idiomatisierten, ,metaphorischen' Raumausdrücken fehlten:

(13) (a) dann gehen die wieder nachhause (LO O_JH_1 339)

(b) ohne Geschwister jetzt zuhause hängen (FAB N_JH_2 932)

(c) keiner sagt so jemand in die Fresse (LO O_JH_1 1318 )

(d) so tief ins Detail wollten wir auch nicht gehen (MA N_JH_1 383)

(e) alles in Ordnung (SO N_JH_5 696)

(f) kam mir so in den Sinn (FAB N_JH_2 618)

(g) will er sich jeden Monat tausend Euro zur Seite legen (FAB N_JH_2 1171)

Vermutlich gibt es weitere Einschränkungen, die auf der Basis umfangreicherer Materialsammlungen beschrieben werden müssen. Typische grammatische Kontexte, in denen die Präposition fehlt, sind hingegen die Verben gehen oder sein oder die Existenzpartikel gibts, deren semantische Rahmenstruktur (frame) bereits eine Lokalisierung oder Richtung enthält ${ }^{12}$ (vgl. Wiese/ Duda ersch.demn.):

$12 \quad$ Als zweiwertige Partikel selegiert gibts einen Lokalisierungsausdruck. 
(14) (a) gehen Sie andere stadtteil (LO O_JH_1 623)

(b) Wenn man Wasen geht... (MA N_JH_1 1405)

(c) ich geh doch Puff (FU N_JH_1 1250)

(d) dann musst ich Rathaus mit (ES N_RS_1 1519)

(e) hier Ost gibts keine Mädchens (LO O_JH_1 1382)

(f) früher war ich Verein (ER BC_JA_08 504)

(g) Bushido war Saturn (MV BC_JA_02 329)

Manche Nominalphrasen, die im autochthonen Deutsch präpositional markiert werden müssten, werden in der Sprache der Jugendlichen als freie Themen (ohne Präposition) vor den Satz gestellt. Ethnisch markiert ist hier vermutlich weniger die präpositionslose NP selbst, als die mangelnde Wiederaufnahme dieses freien Themas durch ein Pronomen im nachfolgenden Satz. Das Fehlen bestimmter anaphorischer Pronomina ist auch über diese Konstruktion hinaus ein wichtiges Merkmal der Syntax der Jugendlichen. In den folgenden Beispielen ist die zu erwartende, aber nicht realisierte pronominale Struktur in [ ] ergänzt:

(15) (a) also die ersten beiden Schwestern, die älteren, [mit denen] hab ich mich sehr gut verstanden (MA2 N_JH_2 935)

(b) Kvick, [da] sind halt viele so so Mädchens drinne (MV BC_JA_01 517)

(c) also meine Klasse zum Beispiel, [da] sin die meisten Deutsche (FAB N_JH_2 307)

(d) Spanien und Italien, [da] kannst du dir jedes Auto holen (FAB N_JH_2 1209)

Berücksichtigt man lediglich Fälle, in denen die unter (11)-(13) exemplifizierten Beschränkungen für präpositionslose Phrasen eingehalten werden, steigt deren Prozentsatz erheblich an, nämlich von 9,4\% auf $25 \%$ aller Belege.

\subsubsection{Nicht-Verwendung des indefiniten Artikels}

Ein zweites wichtiges syntaktisches Merkmal ist das Fehlen des indefiniten Artikels. Der indefinite Artikel unterliegt auch im autochthonen Deutsch Schwächungen und Tilgungen. Die im gesprochenen Deutsch fast obligatorische Klitisierung von ein $>n$ führt insbesondere in den hochfrequenten Verbindungen ein bisschen und ein paar oft zum völligen Ausfall; beide werden bereits vielfach nicht mehr als $n \_b i s s c b e n$ und $n$ _paar, sondern als bisschen und 
paar realisiert. In der Auswertung in Tabelle 2 wurden diese Formen deshalb nicht berücksichtigt. Es fehlen dann in 53 von insgesamt 304 Fällen die indefiniten Artikel (17\%). Durchschnittlich realisiert ein Sprecher in 12,9\% der im autochthonen Deutsch üblichen Fälle den indefiniten Artikel nicht.

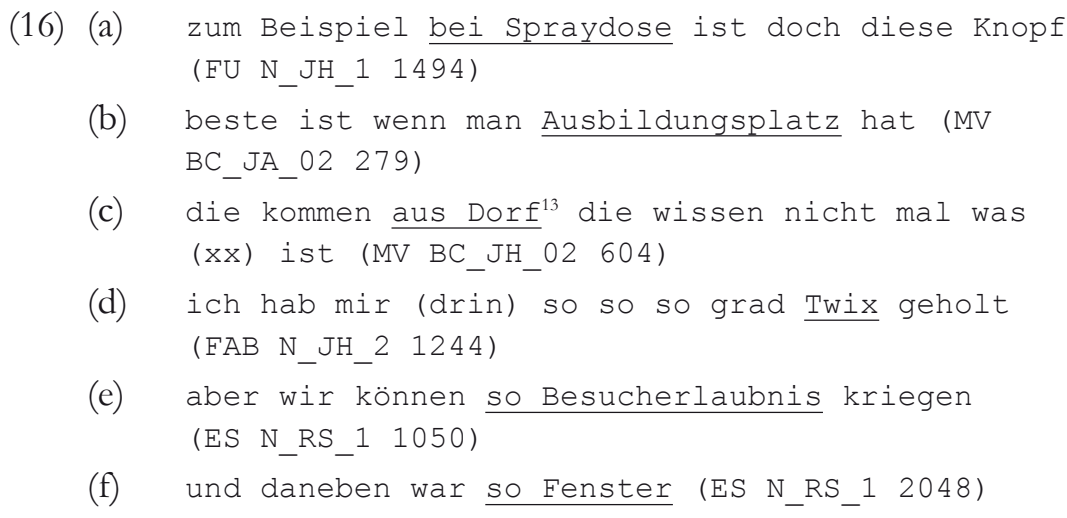

Da der Artikel im Deutschen oft die einzige overte Kasusmarkierung in der NP trägt, führt der Verzicht auf den Artikel dann auch zu oberflächlich kasuslosem Sprechen. Ob die Kategorie Kasus überhaupt noch relevant ist, lässt sich entscheiden, wenn trotz fehlenden Artikels ein modifizierendes Adjektiv auftritt. In einem solchen Fall wird teils der Kasus am Adjektiv markiert (vgl. $(17 \mathrm{a}-\mathrm{c})$, teils wird eine neutrale Form des modifizierenden Adjektivs gewählt, die aus dem Suffix /-e/ ${ }^{14}$ besteht (vgl. Bsp. (d)-(f)). In diesem zweiten Fall gibt es keine Evidenz mehr dafür, dass die grammatische Kategorie des Kasus noch vorhanden ist.
(17) (a) hat der falsches Wort gesagt... (LO O_JH_1 935)
(b) und die haben dann halt so ganz dünnen stift... (FU N_JH_1 1485)
(c) einmal halbes Jahr lang (ER BC_JA_08 442)
(d) die haben komische Dialekt (MV BC_JA_02 14)
(e) die ham dort gute Abschluss oder so (MA2 N_JH_2 2094)
(f) hast deutsche Pass? (SO N_JH_5 1168)

Ob Kasus hier mit Genus interagiert, muss genauer untersucht werden.

13 Nicht immer ist entscheidbar, ob der fehlende Artikel definit oder indefinit zu sein hätte, so in diesem Beispiel.

14 Die alternative Erklärung, dass die Schwa-Endungen ein Resultat der im Alemannischen verbreiteten Tilgung des $/ \mathrm{n} /$ in unbetonten auslautenden /en/-Silben ist, erscheint angesichts der sonst fehlenden Dialekteinflüsse unwahrscheinlich. 


\subsubsection{Nicht-Verwendung des definiten Artikels}

Wie aus Tabelle 2 ersichtlich, werden auch die im autochthonen Deutsch geforderten definiten Artikel in einem Teil der Belege nicht gesetzt. ${ }^{15}$ Die durchschnittliche Anzahl der NPs ohne definiten Artikel pro Sprecher liegt mit 16,1\% etwas über der durchschnittlichen Anzahl der NPs ohne indefiniten Artikel (12,9\%). Allerdings korrelieren die Werte nicht, d.h. ein Sprecher, der vergleichsweise häufig die indefiniten Artikel weglässt, muss keine vergleichsweise hohen Werte für Nominalphrasen ohne definite Artikel haben und umgekehrt. Einflüsse der Familiensprache sind nicht erkennbar; die Sprecher mit romanischem Sprachhintergrund (MA, FAB, MA2) verwenden den definiten Artikel etwa genauso oft nicht wie die Sprecher mit türkischem (FU, SO, BU, ER, MV) oder slavischem (LO) Familienhintergrund. Hier einige Beispiele (die relevanten NPs sind unterstrichen):

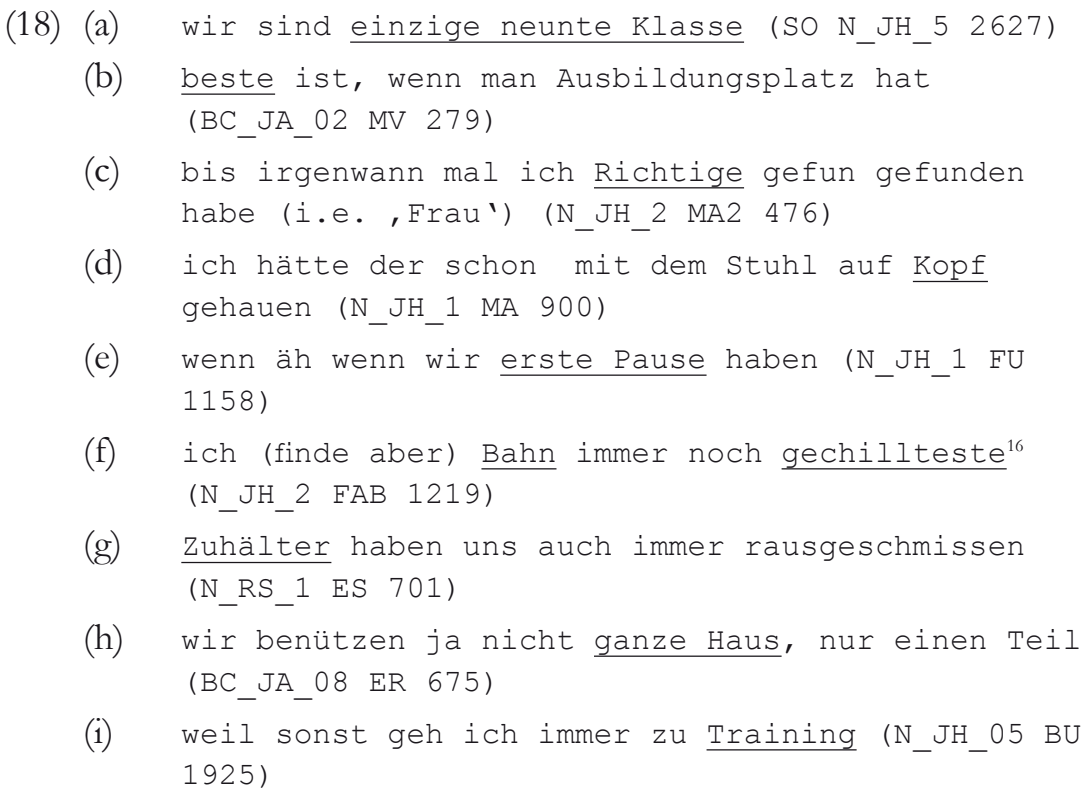

In vielen Kontexten ist der definite Artikel im Deutschen arbiträr und funktionslos, etwa wenn er vor Quantoren oder Superlativen steht (vgl. (a, b, f)), bei Unika (f), oder wenn durch den grammatischen Kontext eindeutig defi-

\footnotetext{
15 Gezählt wurden hier nur die NPs mit definitem Artikel, und zwar einschließlich der klitischen und mit Präpositionen verschmolzenen Artikel. Es gibt natürlich andere definite Nominalphrasen, die durch possessive oder demonstrative (dieser) Artikelformen markiert werden. Sie sind hier nicht berücksichtigt.

16 Von chillen, entspannen', gechillt, entspannt'.
} 
nierte Elemente des semantischen Rahmens markiert werden (d). Es ist also nicht verwunderlich, dass sich hier alternative, artikellose Konstruktionsmuster etablieren können.

Unsere Sprecher zeigen systematisch auch andere Merkmale, die teils auch von Wiese $(2006,2009,2012)$ in Berlin festgestellt worden sind. Selten kommen Verletzungen der XV-Struktur des Deutschen vor. Häufiger ist das Fehlen bestimmter anaphorischer Pronomina sowie des suppletiven Pronomens es, mit dem die Reanalyse von gibts als nicht-analyiserbarer Partikel zusammenhängt. Die Rekurrenz der Merkmale erlaubt es durchaus auch in der Grammatik, von einem (Poly-)Ethnolekt zu sprechen. Diese Beschreibung wäre allerdings unvollständig, wenn man nicht darauf hinweisen würde, dass es in der Sprache unserer Informanten auch eine erhebliche Anzahl von vermutlich unsystematischen Abweichungen von der autochthonen Grammatik des Deutschen gibt, die auf eine unsichere Beherrschung der deutschen Morphologie hindeuten, aber nicht ohne Weiteres als Evidenz für die Herausbildung einer neuen, systemhaften Varietät dienen können. Einige Beispiele für unsystematische Kongruenz- und Rektionsfehler sind die folgenden (alle von Sprecher BU):

(19) (a) wir werden nicht wie die Deutsche rausgeschmissen
(b) ich werd nie ein deutsche Pass bekommen
(c) mein Mutter ned
(d) mein persönlich Meinung
(e) ich tolerier halt die Schwule
(f) jeden Mensch ist Mensch, Mann!
(g) wir schlafen zusammen in ein Bett

Möglicherweise restrukturiert sich das System dieses Sprechers durch Wegfall der Endungen (v.a. in der Artikelform) bzw. durch Verwendung einer passe-partout-Endung /-e/ (realisiert als Schwa). Ob dahinter allgemeinere Strukturentwicklungen stehen, muss noch untersucht werden.

\section{Schlussbemerkungen}

Die (poly-)ethnischen grammatischen Merkmale der hier untersuchten Stuttgarter Jugendlichen vereinfachen das allochthone Deutsche ohne Frage. Sie geben grammatische Komplexität allerdings vor allem dort auf, wo sie funktional nicht nötig ist. Das geschieht auf systematische Art und Weise, wenn auch nur in einem kleinen Teil der möglichen Fälle. Dass die in Stuttgart gefundenen grammatischen Merkmale der Weglassung von Artikelformen und Präpositionen auch in Berlin und Hamburg nachgewiesenen werden 
können, belegt außerdem ihre überregionale Reichweite. Sind die grammatischen Merkmale des ,Kiezdeutsch deshalb aber, wie Wiese argumentiert, „typisch deutsch“ (2012, S. 48) und lediglich ,in anderen Dialekten oder im Standard (noch) nicht realisiert" (ebd., S. 36 f.)? Muss die neue, (poly-) ethnolektale Varietät also als ebenso deutsch gelten „wie das Bairische“ und ist sie „ebenso in Grammatik, Wortschatz und Aussprache fest im System des Deutschen verankert" (ebd., S. 206)? Die diskutierten grammatischen Neuerungen (sowie auch das hier nicht behandelte Fehlen der obligatorischen anaphorischen und der suppletiven Pronomina sowie Veränderungen der XV-Stellung) greifen tief in die Strukturen des autochthonen Deutschen in seiner Standard- wie seinen Non-Standardformen ein. Zwar sind sie (mit Ausnahme der Präpositionen) aus typologischer Sicht markiert und kommen nur in verhältnismäßig wenigen Sprachen der Welt vor (besonders gilt das für die auf germanische Sprachen eingeschränkte XV-Stellung). Es verwundert daher nicht, dass Lernervarietäten des Deutschen sowie Kontaktvarietäten ähnliche strukturelle Veränderungen zeigen. Dass es hier um ,verletzliche'Strukturen geht, bedeutet aber nicht, dass sie nicht für das Deutsche in einer grundlegenden Weise typisch wären. Eine Varietät des Deutschen ohne Artikelformen und Lokal-/Direktionalpräpositionen, mit SVO- anstelle von XV-Stellung, ohne obligatorische Subjektfüllung durch suppletive Pronomina und ohne obligatorische Anaphern wäre sicher nicht mehr „fest im System des [heutigen autochthonen] Deutsch verankert".

Aber ist es wahrscheinlich, dass sich eine solche Varietät unter den gegebenen gesellschaftlichen Bedingungen stabilisieren wird? Die Zeiten sind nicht gut für die Entstehung und Verfestigung neuer Varietäten. In spätmodernen Gesellschaften sind die Sprecher Selbst-Stilisierer, sie verorten sich in einer schnell veränderbaren sozialen Welt, in der semiotische Mittel ständig neu zusammengesetzt werden, um soziale Stile zu schaffen. Besonders Jugendliche sind Meister dieser Bricolage. Varietäten im Sinne von sprachlichen Systemen, die durch interne Kookkurrenzen und vielleicht auch soziale und politische Instanzen stabil gehalten werden, erfordern Zeit, um sich stabilisieren zu können. Es ist unwahrscheinlich, dass diese Stabilisierung im vorliegenden Fall eintreten wird. Eher als das Entstehen von Varietäten beobachten wir nämlich, dass existierende Varietäten als Steinbrüche für die sprachlichen Versatzstücke dienen, die in soziale Stile inkorporiert werden. Die deutschen Dialekte sind dafür ein gutes Beispiel: Sie sind mehr und mehr nur noch Ressourcen, auf die die Sprecher zurückgreifen, um regionale Stile zu schaffen. Diese sind in der Regel standardnah und nutzen nur noch selektiv dialektale Merkmale, um regionale Identitäten auszudrücken.

,Kiezdeutsch', ,Straßensprache' oder, Ghettodeutsch' sind ebenfalls in erster Linie Bezeichnungen für eine Ansammlung von solchen sozialen Stilen, die von Jugendlichen geformt, transformiert und wieder aufgelöst werden. Sie sind mit einer Selbst- und Fremdkategorisierung als Außenseiter 
der Gesellschaft - ,Ausländer ${ }^{`}$ im Sinne der Sprecher selbst - verbunden. Insofern ist auch keine Romantisierung angebracht: Die Selbstpositionierung, die die Jugendlichen damit herstellen, verspricht weder Bildungserfolg noch berufliche Karriere. Allerdings wissen das die Jugendlichen auch selbst, und ganz im Sinn spätmoderner Gesellschaften haben sie in der Regel mehrere sprachlich-soziale Identitäten in ihrem Repertoire, die sie situationsspezifisch einsetzen.

Für die Selbst-Stilisierung als Ghetto-Jugendlicher reichen oft schon wenige sprachliche Merkmale aus. Dennoch ist es richtig, dass dieselben sprachlichen Merkmale zusammen genommen alles Zeug dazu hätten, eine neue Varietät zu konstituieren, die wesentlich vom autochthonen Deutsch abweichen würde. Diese Varietät wird aber bisher von niemand konsistent gesprochen; und damit sie sich konsolidieren könnte, bedürfte es einer massiven sozialen Segregation ihrer Sprecher und Sprecherinnen, die trotz aller Diskussionen um, Parallelgesellschaften' im heutigen Deutschland nicht gegeben ist. Der (Poly-)Ethnolekt ist daher einstweilen nur ein Destillat der Linguisten und Linguistinnen aus den verwendeten stilistischen Ressourcen. Dieses Destillat ist es aber allemal wert, empirisch umfassend analysiert zu werden.

\section{Literatur}

Agha, Asif (2003): The social life of cultural value. In: Language \& Communication 23, S. 231-273.

Agha, Asif (2007): Language and social relations. (= Studies in the Social and Cultural Foundations of Language 24). Cambridge.

Androutsopoulos, Jannis (2007): Ethnolekte in der Mediengesellschaft. Stilisierung und Sprachideologie in Performance, Fiktion und Metasprachdiskurs. In: Fandrych, Christian/Salverda, Reinier (Hg.): Standard, Variation und Sprachwandel in germanischen Sprachen. (= Studien zur Deutschen Sprache 41). Tübingen, S. 113-157.

Auer, Peter (1981): Zur indexikalitätsmarkierenden Funktion der demonstrativen Artikelform in deutschen Konversationen. In: Hindelang, Götz (Hg.): Sprache. Verstehen und Handeln. (= Akten des Linguistischen Kolloquiums 15,2; Linguistische Arbeiten 99). Tübingen, S. 301-311.

Auer, Peter (2003): ,Türkenslang ${ }^{6}$ - ein jugendsprachlicher Ethnolekt des Deutschen und seine Transformationen. In: Häcki Buhofer, Annelies (Hg.): Spracherwerb und Lebensalter. (= Basler Studien zur deutschen Sprache und Literatur 83). Tübingen, S. 255-264.

Auer, Peter (2006): Sociolinguistic crossing. In: Brown, Keith (Hg.): Encyclopedia of language and linguistics. Bd. 11. 2. Aufl. Amsterdam, S. 490-492.

Benor, Sarah B. (2010): Ethnolinguistic repertoire: shifting the analytic focus in language and ethnicity. In: Journal of Sociolinguistics 14, S. 159-183. 
Blommaert, Jan (2010): The sociolinguistics of globalization. (= Cambridge Approaches to Language Contact). Cambridge.

Cheshire, Jenny et al. (2011): Contact, the feature pool and the speech community: the emergence of multicultural London English. In: Journal of Sociolinguistics 15, S. 151-196.

Clyne, Michael (2000): Lingua franca and ethnolects in Europe and beyond. In: Sociolinguistica 14, S. 83-89.

Deppermann, Arnulf (2007): Playing with the voice of the other. Stylized Kanaksprak conversations among German adolescents. In: Auer, Peter (Hg.): Style and social identities. (= Language, Power and Social Process 18). Berlin, S. 325-361.

Dirim, İnci/Auer, Peter (2004): Türkisch sprechen nicht nur die Türken. Über die Unschärfebeziehung zwischen Sprache und Ethnie in Deutschland. (= Linguistik Impulse \& Tendenzen 4). Berlin.

Eckert, Penelope (2000): Linguistic variation as social practice. The linguistic construction of identity in Belten High. (= Language in Society 27). Oxford.

Eckert, Penelope (2004): The meaning of style. In: Texas Linguistic Forum 47, S. 4153.

Freywald, Ulrike et al. (2011): Kiezdeutsch as a multiethnolect. In: Kern/Selting (Hg.), S. 45-74.

Füglein, Rosemarie (2000): Kanak Sprak. Eine ethnolinguistische Untersuchung eines Sprachphänomens in Deutschland. Diplomarbeit, Fakultät für Sprach- und Literaturwissenschaften der Otto-Friedrich-Universität Bamberg (unveröffentlicht).

Keim, Inken (2007): Die „türkischen Powergirls“. Lebenswelt und kommunikativer Stil einer Migrantinnengruppe in Mannheim. (= Studien zur Deutschen Sprache 39). Tübingen.

Kern, Friederike (2011): Rhythm in Turkish German talk-in-interaction. In: Kern/Selting (Hg.), S. 161-190.

Kern, Friederike/Selting, Margret (Hg.) (2011): Ethnic styles of speaking in European metropolitan areas. (= Studies in Language Variation 8). Amsterdam/Philadelphia.

Madsen, Lian Malai (2011): Late modern youth style in interaction. In: Kern/Selting (Hg.), S. 265-290.

Quist, Pia (2008): Sociolinguistic approaches to multiethnolect: language variety and stylistic practice. In: International Journal of Bilingualism 12, S. 43-61.

Quist, Pia (2010): Untying the language-body-place connection: A study on linguistic variation and social style in a Copenhagen community of practice. In: Auer, Peter/ Schmidt, Jürgen Erich (Hg.): Language and space. An international handbook of linguistic variation. Bd. 1: Theories and methods. (= Handbücher zur Sprach- und Kommunikationswissenschaft 30). Berlin/New York, S. 632-648.

Rampton, Ben (1995): Crossing. Language and ethnicity among adolescents. (= Real Language Series). London.

Rampton, Ben (2011): From ,Multi-ethnic adolescent heteroglossia' to ,Contemporary urban vernaculars'. In: Language \& Communication 31, S. 276-294. 
Sharma, Devyani (2011): Style repertoire and social change in British Asian English. In: Journal of Sociolinguistics 15, S. 464-492.

Tertilt, Hermann (1996): Turkish Power Boys. Ethnographie einer Jugendbande. Frankfurt a.M.

Vertovec, Steven (2007): Super-diversity and its implications. In: Ethnic and Racial Studies 30, S. 1024-1054.

Wenger, Etienne (1998): Communities of practice: learning, meaning, and identity. (= Learning in Doing). Cambridge.

Wiese, Heike (2006): „Ich mach dich Messer“. Grammatische Produktivität in der Kiez-Sprache. In: Linguistische Berichte 207, S. 245-273.

Wiese, Heike (2009): Grammatical innovation in multiethnic urban Europe: new linguistic practices among adolescents. In: Lingua 119, S. 782-806.

Wiese, Heike (2010): Kiezdeutsch. In: Aus Politik und Zeitgeschehen 8, S. 33-38.

Wiese, Heike (2011): So as a focus marker in German. In: Linguistics 49, S. 991-976.

Wiese, Heike (2012): Kiezdeutsch - ein neuer Dialekt entsteht. München.

Wiese, Heike/Duda, Sibylle (ersch.demn.): A new German particle gib(t)s: The dynamics of a successful cooperation. In: Spalek, Katharina/Domke, Juliane/Grommes, Patrick (Hg.): Erwerb und Kontext: Beiträge zu sprachlichen Vergleichen, Varietäten, Variationen. Tübingen. 